\title{
Hydrodynamic interactions among multiple circular cylinders in an inviscid flow
}

\author{
R. $\operatorname{Sun}^{1} \dagger$ and C. O. $\mathrm{Ng}^{2}$ \\ ${ }^{1}$ Department of Engineering Mechanics, Shanghai Jiao Tong University, Shanghai 200240, China \\ 2 Department of Mechanical Engineering, The University of Hong Kong, Pokfulam, Hong Kong, China
}

(Received 9 December 2011; revised 3 June 2012; accepted 29 August 2012;

first published online 9 October 2012)

Hydrodynamic interactions among multiple circular cylinders translating in an otherwise undisturbed inviscid fluid are theoretically investigated. A constructive method for solving a Neumann boundary-value problem in a domain outside $N$ circles (one kind of Hilbert boundary-value problem in the complex plane) is presented in the study to derive the velocity potential of the liquid. The method employs successive offset functions combined with a 'generalized cyclic permutation' in turn to satisfy the impenetrable boundary condition on each circle. The complex potential is therefore expressed as $N$ isolated singularities in power series form and used to get instantaneous added masses of $N$ submerged circular cylinders. Then, based on the Hamilton variational principle, a dynamical equation of motion in vector form is derived to predict nonlinear translations of the submerged bodies under fully hydrodynamic interactions. Also, the equivalence of the energy-based Lagrangian framework and a momentum-type one in the two-dimensional body-liquid system is proved. It implies that the pressure integration around a submerged body is holographic, which provides information about velocities and accelerations of all bodies. The numerical solutions indicate some typical dynamical behaviours of more than two circular cylinders which reveal that interesting nonlinear phenomena would appear in such a system with simple physical assumptions.

Key words: general fluid mechanics, Hamiltonian theory, mathematical foundations

\section{Introduction}

The hydrodynamic interaction between multiple submerged moving bodies is of fundamental importance for understanding of unsteady motions of the bodies (Lamb 1932; Milne-Thomson 1960). Usually, to make the problem treatable, the potential flow theory of incompressible fluids is employed since it provides a good approximation for high-Reynolds-number flows. However, the physical mechanism behind the dynamical behaviours of the bodies has not been satisfactorily explored up to now owing to the difficult mathematical treatment for the corresponding problem in the multiple connected regions.

The hydrodynamic interaction between many cylinders immersed in an unbounded inviscid liquid was first addressed by Hicks (1879), who studied the impact of 
two circular cylinders. Yamamoto (1976) explored the problem of two circular cylinders translating arbitrarily in the flow by continually using the Milne-Thomson circle theorem to satisfy the boundary condition on each surface. To break through the restriction of the body geometry, Landweber \& Chwang (1989) developed a boundary-integral model for two-body interaction problems by generalizing the Taylor (1928) formula. Using numerical modelling, Landweber, Chwang \& Guo (1991) investigated oblique translations of two two-dimensional bodies. Taking advantage of twin multipole expansions only at two isolated points and successive images of a closed form, Sun \& Chwang $(2000,2006)$ presented a method of successive offset functions to derive velocity potentials for two simple geometries, and explored the hydrodynamic translation-rotation coupling between the non-circular bodies. To motivate the approach, two sets of transformations between 'body shape' harmonics need to be established in two body-fixed coordinate systems; as a result, each velocity potential is composed of twin singularities in harmonic series form, and their coefficients are reduced to iterative formulae. These make the velocity potential suitable for hydrodynamic analysis and rapid calculation of added masses and their spatial derivatives.

In recent years there has been a revival of interest in investigation of the hydrodynamic interaction due to planar motions of cylinders in an inviscid liquid. Wang (2004) contributed to this specific problem with an extension to two expanding and translating circular cylinders. The corresponding velocity potential is derived using a conformal mapping and Fourier series. Almost at the same time, Burton, Gratus \& Tucker (2004) studied the same problem from a different mathematical viewpoint. Crowdy, Surana \& Yick (2007) and Tchieu, Crowdy \& Leonard (2010) addressed the planar issues of two complicated geometries using the Villat formula and the Laurent series expansion, respectively. The velocity potentials are expressed as contour integrals on two concentric circles which are mapped using the Möbius transformation from the two arbitrarily shaped bodies. Especially to resolve the problem involving more two-dimensional bodies, Crowdy (2010) developed a new approach for finding the complex potentials associated with their motion in a liquid. The key to the generality of the approach is the use of the Riemann mapping theorem together with a special transcendental function called the Schottky-Klein prime function. From the method, he obtained the complex potentials for a uniform flow past multiple cylinders (Crowdy 2006), for an arbitrary collection of moving cylinders (Crowdy 2008) and for two-dimensional bodies of arbitrary shape with point vortices (Crowdy 2010). These velocity potentials for liquid with many submerged moving cylinders are usually expressed as contour integrals and may be solved using the boundary-element method. Furthermore, the two-dimensional exact solution for circular cylinders has been applied to more practical problems. Wang $(2005,2007)$ studied the irrotational flow induced by slender bodies in very close proximity. He divided the flow field into an outer region far away from the two bodies and an inner region near them, and analysed it using the method of matched asymptotic expansions.

Generally, there are two ways of describing translations and rotations of bodies in a liquid. One is concerned with a momentum-type framework, where the hydrodynamic loads are simply determined by integrating the pressure around each body surface (cf. Landweber \& Yih 1956; Cummins 1957; Landweber \& Miloh 1980; Wang 2004; Crowdy et al. 2007; Tchieu et al. 2010). The other involves an 
energy-based Lagrangian framework, which includes two sets of differential equations derived from the Hamilton variational principle: Kelvin-Kirchhoff's equations of motion in a relative frame of reference and Lagrange's equations in the absolute reference frame (see Lamb 1932). The equivalence between the two sets of equations was proved by Miloh \& Landweber (1981). Although the energy-based Lagrangian framework is believed to be equivalent to the momentum-type one in the hydrodynamic community, the connection between them has not yet been proved. This energy-based approach reveals that accelerations of the submerged bodies are coupled with one another by their added masses and hydrodynamic forces determined by spatial derivatives of the added masses. Accordingly, it is adopted that dynamic behaviours of many submerged simple bodies are described under a fully hydrodynamic interaction (cf. Guo \& Chwang 1991; Landweber et al. 1991; Sun \& Chwang 2000, 2006; Nair \& Kanso 2007).

At the present time, the powerful modern computational capabilities allow us to assess the hydrodynamic interaction among multiple bodies of arbitrary shape in unsteady-flow surroundings. However, it remains highly desirable to be able to predict and explain the main features behind the physical modelling; clearly the numerical procedure does not provide the whole answer. Therefore, to perform a theoretical prediction of the hydrodynamic forces on many submerged circular cylinders and their nonlinear motions, the investigation develops an approach for constructing analytic expressions for the velocity potential of the liquid with these cylinders so as to derive the added masses and their spatial derivatives in closed form. To this end, an operation involving successive offset functions combined with a 'generalized cyclic permutation' is introduced. In this manner, the corresponding complex potential may be expressed as $N$ singularities in power series form, and thus added masses and their spatial derivatives are composed of those series whose terms decay by the order $O\left(s^{-2}\right)$, where $s$ is the centre-to-centre distance between two relevant circular cylinders, and readily reduced to recurrence formulae so as to be suitable for the hydrodynamic analysis. In addition, a dynamical equivalence of the energybased Lagrangian framework and momentum-type framework in two dimensions is proved. Subsequently, some examples are discussed so as to explore the nature of the hydrodynamic interaction among many bodies.

\section{Formulation of the problem and its solution}

Let us consider a planar translation of $N(N \geqslant 2)$ circular cylinders in an unbounded fluid with their axes perpendicular to the plane of motion, which has absolute Cartesian coordinates $\left(x_{1}, x_{2}\right)$. In the two-dimensional frame of reference, circle $\alpha(\alpha \in\{1, \ldots, N\})$ (indicating cylinder $\alpha)$ of radius $R_{\alpha}$ and with centre $o_{\alpha}$ (at $\left.\boldsymbol{x}_{\alpha}=\left(x_{\alpha 1}, x_{\alpha 2}\right)\right)$ moves at velocity $\boldsymbol{u}_{\alpha}\left(\boldsymbol{u}_{\alpha}=\left(u_{\alpha 1}, u_{\alpha 2}\right)\right)$, and its boundary is denoted by $c_{\alpha}$, as shown in figure 1 .

The fluid is assumed to be incompressible and inviscid, and the flow to be irrotational. Hence, there exists a velocity potential $\varphi$ which may be expressed as

$$
\phi=\boldsymbol{u}_{\alpha} \cdot \boldsymbol{\varphi}_{\alpha}=u_{\alpha i} \varphi_{\alpha i} \quad(\alpha=1, \ldots, N ; i=1,2),
$$

where a repeated index indicates a summation. Here $\boldsymbol{\varphi}_{\alpha}$ denotes the unit velocity potential vector due to the motion of circle $\alpha$, and $\varphi_{\alpha i}$ its $i$ th component corresponding to the $i$ th velocity component $u_{\alpha i}$. The kinematic boundary condition on circle $\alpha$ is 


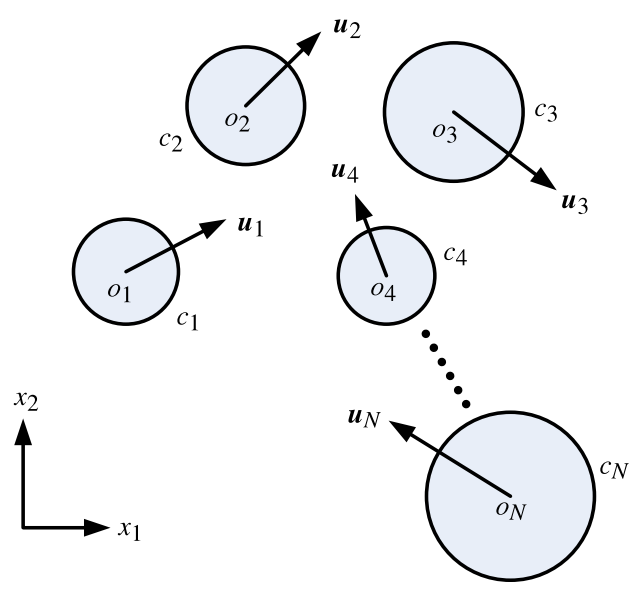

FIGURE 1. Sketch of $N$ planar-translating circular cylinders.

given by

$$
\left.\boldsymbol{n}_{\alpha} \cdot \nabla \phi=\boldsymbol{n}_{\alpha} \cdot \boldsymbol{u}_{\alpha} \quad \text { (not summed for } \alpha ; \alpha \in\{1, \ldots, N\}\right),
$$

where $\boldsymbol{n}_{\alpha}$ is a unit outward normal vector to the boundary of circle $\alpha$. And thus the unit potential $\varphi_{\alpha i}$ should satisfy the boundary conditions

$$
\partial \varphi_{\alpha i} / \partial n_{\beta}=\delta_{\alpha \beta} n_{\beta i} \quad(\text { not summed for } \beta ; \beta \in\{1, \ldots, N\}),
$$

where $n_{\beta}$ denotes distance along an outward normal to circle $\beta, \delta_{\alpha \beta}$ the Kronecker delta, and $n_{\beta i}$ the $i$ th component of $\boldsymbol{n}_{\beta}$.

For the above two-dimensional Hamilton system, the complex variable $z$ is adopted and as usual defined to be $x_{1}+\mathrm{i} x_{2}$ so as to solve the corresponding Neumann boundaryvalue problem. This problem can be regarded as one kind of Hilbert boundary-value problem in the complex plane. The problem is stated as follows: suppose $D$ is an $N+1$ connected domain in the complex plane $Z$, and its boundary is $\partial D=\sum_{\alpha=1}^{N} c_{\alpha}$, where $c_{\alpha}$ indicates circle $\alpha$; find a continuous solution $w(z)$ in $D$ which satisfies the boundary conditions

$$
\operatorname{Re}\left[n(z) \frac{\mathrm{d} w(z)}{\mathrm{d} z}\right]=\operatorname{Re}[\bar{n}(z) V(z)], \quad z \in \partial D,
$$

where $n(z)$ denotes a complex unit normal, and $V(z)$ a given boundary function. In this paper, a variable with an overbar indicates its complex conjugate.

Solutions satisfying boundary condition (2.3) can be obtained using a constructive method which is shown in the Appendix. Based on the solutions, when a single circle $\alpha(\alpha \in\{1, \ldots, N\})$ of radius $R_{\alpha}$ translates at an arbitrary velocity $\left(u_{\alpha 1}, u_{\alpha 2}\right)$ through an unbounded liquid, the complex potential of the liquid is

$$
w_{\alpha}=-\left(u_{\alpha 1}+\mathrm{i} u_{\alpha 2}\right) R_{\alpha}^{2} / z_{\alpha}^{\prime} \quad(\text { not summed for } \alpha),
$$

where $z_{\alpha}^{\prime}$ denotes the complex variable in auxiliary complex plane $Z_{\alpha}^{\prime}$. From the derivation in the Appendix, the complex potential in the case of $N$ circles moving 
through a liquid is

$$
\begin{aligned}
w= & -u_{\alpha j} R_{\alpha}^{2} w_{\alpha j 1}=u_{\alpha 1}\left\{-R_{\alpha}^{2}\left[\frac{1}{z_{\alpha}^{\prime}}+\sum_{\gamma=1}^{N} \sum_{m=1}^{\infty} \frac{1}{z_{(\alpha+\gamma)^{\dagger}}^{\prime m}} \sum_{i=0}^{\infty} \lambda_{\alpha 1 m 1}^{(N i+\gamma)}\right]\right\} \\
& +u_{\alpha 2}\left\{-\mathrm{i} R_{\alpha}^{2}\left[\frac{1}{z_{\alpha}^{\prime}}+\sum_{\gamma=1}^{N} \sum_{m=1}^{\infty} \frac{1}{z_{(\alpha+\gamma)^{\dagger}}^{\prime m}} \sum_{i=0}^{\infty} \lambda_{\alpha 2 m 1}^{(N i+\gamma)}\right]\right\} \\
= & u_{\alpha j} W_{\alpha j} \quad(\alpha=1, \ldots, N ; j=1,2),
\end{aligned}
$$

where $W_{\alpha j}$ is a unit complex potential corresponding to $u_{\alpha j}$.

\section{Added-mass tensor and dynamical equations of motion}

If there is no point vortex and the cylinders are not deformable, the kinetic energy $T_{f}$ of the liquid is integrable in the unbounded region, and may be expressed as

$$
T_{f}=-\frac{\rho}{2} \int_{\Sigma c_{\beta}} \phi \frac{\partial \phi}{\partial n_{\beta}} \mathrm{d} l_{\beta} \quad(\beta=1, \ldots, N),
$$

where $l_{\beta}$ denotes a curvilinear distance along circle $\beta$ and $\rho$ the liquid density and $\Sigma c_{\beta}$ denotes the sum of all circles.

For convenience, let us establish an expanded $2 N$-dimensional Cartesian space, in which generalized position, velocity and unit velocity potential vectors are defined as

$$
\boldsymbol{X}=x_{\alpha j} \boldsymbol{e}_{2(\alpha-1)+j}, \quad \boldsymbol{U}=u_{\alpha j} \boldsymbol{e}_{2(\alpha-1)+j}, \quad \boldsymbol{\phi}=\varphi_{\alpha j} \boldsymbol{e}_{2(\alpha-1)+j} \quad(\alpha=1, \ldots, N ; j=1,2),
$$

where $\boldsymbol{e}_{i}$ is unit vector $i$ of the expanded space.

Therefore, $\phi=\boldsymbol{U} \cdot \boldsymbol{\phi}=\boldsymbol{\phi} \cdot \boldsymbol{U}$ from (2.1), and then

$$
T_{f}=-\frac{\rho}{2} \int_{\Sigma c_{\beta}} \phi \frac{\partial \phi}{\partial n_{\beta}} \mathrm{d} l_{\beta}=-\frac{\rho}{2} \int_{\Sigma c_{\beta}} \boldsymbol{U} \cdot \boldsymbol{\phi} \frac{\partial \boldsymbol{\phi}}{\partial n_{\beta}} \cdot \boldsymbol{U} \mathrm{d} l_{\beta}=\frac{1}{2} \boldsymbol{U} \cdot \boldsymbol{A} \cdot \boldsymbol{U},
$$

where

$$
\boldsymbol{A}=-\rho \int_{\Sigma c_{\beta}} \boldsymbol{\phi} \frac{\partial \boldsymbol{\phi}}{\partial n_{\beta}} \mathrm{d} l_{\beta}
$$

is a tensor since $\boldsymbol{\phi} \partial \boldsymbol{\phi} / \partial n_{\beta}$ indicates a dyadic of two vectors, $\boldsymbol{\phi}$ and $\partial \boldsymbol{\phi} / \partial n_{\beta}$, representing $2 N \times 2 N$ instantaneous added masses which exhibit a non-uniform distribution in space. It is easy to prove, from Green's reciprocal formula, that $\boldsymbol{A}$ is a symmetric tensor with $N(2 N+1)$ independent instantaneous added-mass coefficients. The coefficient matrix $\left[A_{i, j}\right]$ of instantaneous added-mass tensor $\boldsymbol{A}$ may be obtained by integrating the following matrix expressions over all circles:

$$
\begin{aligned}
& {\left[A_{i, j}\right]=-\rho \int_{\Sigma c_{\beta}}\left[\begin{array}{c}
\varphi_{11} \\
\varphi_{12} \\
\vdots \\
\varphi_{N 1} \\
\varphi_{N 2}
\end{array}\right]\left[\begin{array}{lllll}
\delta_{1 \beta} n_{\beta 1}, & \delta_{1 \beta} n_{\beta 2}, & \cdots & , \delta_{N \beta} n_{\beta 1}, & \delta_{N \beta} n_{\beta 2}
\end{array}\right] \mathrm{d} l_{\beta}} \\
& (\beta=1, \ldots, N) \text {. }
\end{aligned}
$$


With every unit velocity potential $\varphi_{\alpha j}$ one may associate a stream function $\psi_{\alpha j}$. Owing to the relationships $n_{\beta 1} \mathrm{~d} l_{\beta}=\mathrm{d} x_{\beta 2}^{\prime}$ and $n_{\beta 2} \mathrm{~d} l_{\beta}=-\mathrm{d} x_{\beta 1}^{\prime}(\beta=1, \ldots, N)$, where $\mathrm{d} x_{\beta 1}^{\prime}$ and $\mathrm{d} x_{\beta 2}^{\prime}$ are the real and imaginary parts of $\mathrm{d} z_{\beta}^{\prime}$, respectively, it is easy to derive

$$
\rho \int_{c_{\beta}} \psi_{\alpha j} \mathrm{~d} z_{\beta}^{\prime}=-\rho \int_{c_{\beta}} z_{\beta}^{\prime} \delta_{\alpha \beta} n_{\beta j} \mathrm{~d} l_{\beta}=-\delta_{\alpha \beta} B_{\beta} \mathrm{i}^{j-1} \quad(\alpha, \beta \in\{1, \ldots, N\} ; j=1 \text { or } 2),
$$

where $B_{\beta}=\pi \rho R_{\beta}^{2}$ is the mass of the displaced liquid per unit length of cylinder $\beta$. Therefore,

$$
\begin{aligned}
A_{2(\alpha-1)+j, 2 \gamma-1}+\mathrm{i} A_{2(\alpha-1)+j, 2 \gamma}= & -\rho \int_{\Sigma c_{\beta}} \varphi_{\alpha j} \delta_{\gamma \beta} n_{\beta} \mathrm{d} l_{\beta}=\mathrm{i} \rho \int_{c_{\gamma}} \varphi_{\alpha j} \mathrm{~d} z_{\gamma}^{\prime} \\
= & \mathrm{i} \rho \int_{c_{\gamma}}\left(W_{\alpha j}-\mathrm{i} \psi_{\alpha j}\right) \mathrm{d} z_{\gamma}^{\prime} \\
= & \mathrm{i} \rho \int_{c_{\gamma}} W_{\alpha j} \mathrm{~d} z_{\gamma}^{\prime}+\rho \int_{c_{\gamma}} \psi_{\alpha j} \mathrm{~d} z_{\gamma}^{\prime} \\
= & 2 \pi \rho R_{\alpha}^{2} \mathrm{i}^{j-1}\left[\delta_{\alpha \gamma}+\sum_{i=0}^{\infty} \lambda_{\alpha j 11}^{(N i+(N+\gamma-\alpha) \bmod N)}\right] \\
& -\delta_{\alpha \gamma} \pi \rho R_{\alpha}^{2} \mathrm{i}^{j-1} \quad(\alpha, \beta, \gamma \in\{1, \ldots, N\} ; j=1 \text { or } 2) .
\end{aligned}
$$

Similarly, the kinetic energy $T_{b}$ of $N$ circles is

$$
T_{b}=m_{\alpha}\left(u_{\alpha 1}^{2}+u_{\alpha 2}^{2}\right) / 2=\boldsymbol{U} \cdot \boldsymbol{M} \cdot \boldsymbol{U} / 2 \quad(\alpha=1, \ldots, N),
$$

where $m_{\alpha}$ denotes the mass of cylinder $\alpha$ per unit length with density $\rho_{\alpha}$ and

$$
\boldsymbol{M}=m_{\alpha} \boldsymbol{e}_{2(\alpha-1)+j} \boldsymbol{e}_{2(\alpha-1)+j} \quad(\alpha=1, \ldots, N ; j=1,2) .
$$

Since the body-liquid system is conservative, total kinetic energy $T$ of the system should be

$$
T\left(x_{\alpha i}, u_{\alpha i}\right)=T_{f}+T_{b}=\boldsymbol{U} \cdot(\boldsymbol{M}+\boldsymbol{A}) \cdot \boldsymbol{U} / 2 .
$$

On the basis of the Hamilton variational principle expressed as

$$
\delta \int_{t_{i}}^{t_{f}} L \mathrm{~d} t=0
$$

where $t_{i}$ denotes the initial time and $t_{f}$ the end time and $L$ is the Lagrangian function which is equal to the total kinetic energy $T$ in the present case, $T$ should satisfy

$$
\frac{\mathrm{d}}{\mathrm{d} t}\left(\frac{\partial T}{\partial \boldsymbol{U}}\right)-\frac{\partial T}{\partial \boldsymbol{X}}=0
$$

After some tensor manipulations, a dynamical equation of motion in vector form for describing $N$ translational bodies is finally derived as follows:

$$
(\boldsymbol{M}+\boldsymbol{A}) \cdot \frac{\mathrm{d} \boldsymbol{U}}{\mathrm{d} t}=-\boldsymbol{U} \cdot \frac{\mathrm{d} \boldsymbol{A}}{\mathrm{d} \boldsymbol{X}} \cdot \boldsymbol{U}+\frac{1}{2} \frac{\mathrm{d} \boldsymbol{A}: \boldsymbol{U} \boldsymbol{U}}{\mathrm{d} \boldsymbol{X}} .
$$

The first term on the right-hand side of (3.12) indicates an effect arising from spatial non-uniformity of added masses which would statistically exhibit a fluid-momentum transport feature, and the second one expresses an influence of kinetic energy potential of the fluid on the $N$-body movement. Furthermore, it is noted from (3.12) 
that the gradient of the added-mass tensor determines generalized forces acting on the submerged bodies and the tensor itself restricts magnitudes and orientations of accelerations of the bodies. This means the accelerations of all the bodies are coupled with each other.

\section{Equivalence between two frameworks}

Under the same assumptions, the force acting on circle $\alpha, \boldsymbol{F}_{\alpha}(\alpha \in\{1, \ldots, N\})$ in the momentum-type framework can be expressed as a contour integral around the body,

$$
\boldsymbol{F}_{\alpha}=-\int_{c_{\alpha}} p \boldsymbol{n}_{\alpha} \mathrm{d} l_{\alpha}=\int_{c_{\alpha}} \rho \frac{\partial \phi}{\partial t} \boldsymbol{n}_{\alpha} \mathrm{d} l_{\alpha}+\frac{\rho}{2} \int_{c_{\alpha}} \nabla \phi \cdot \nabla \phi \boldsymbol{n}_{\alpha} \mathrm{d} l_{\alpha} \quad(\alpha \in\{1, \ldots, N\}) .
$$

Since the velocity potential $\phi=\phi\left(\boldsymbol{x}, \boldsymbol{x}_{\alpha}(t), \boldsymbol{u}_{\alpha}(t) ; R_{\alpha}\right)$ in the present case, the moving circle $\alpha$ is expressed such that the coordinate $\boldsymbol{x}_{\alpha}$ of its geometric centre varies with time, thus $\int_{c_{\alpha}} \rho(\partial \phi / \partial t) \boldsymbol{n}_{\alpha} \mathrm{d} l_{\alpha}=(\mathrm{d} / \mathrm{d} t) \int_{c_{\alpha}} \rho \phi \boldsymbol{n}_{\alpha} \mathrm{d} l_{\alpha}$, and (4.1a) becomes

$$
\boldsymbol{F}_{\alpha}=-\int_{c_{\alpha}} p \boldsymbol{n}_{\alpha} \mathrm{d} l_{\alpha}=\frac{\mathrm{d}}{\mathrm{d} t} \int_{c_{\alpha}} \rho \phi \boldsymbol{n}_{\alpha} \mathrm{d} l_{\alpha}+\frac{\rho}{2} \int_{c_{\alpha}} \nabla \phi \cdot \nabla \phi \boldsymbol{n}_{\alpha} \mathrm{d} l_{\alpha} \quad(\alpha \in\{1, \ldots, N\}) .
$$

Equation (3.11) may be rewritten as

$$
-\frac{\mathrm{d}}{\mathrm{d} t}(\boldsymbol{A} \cdot \boldsymbol{U})+\frac{\partial T}{\partial \boldsymbol{X}}=\frac{\mathrm{d}}{\mathrm{d} t}(\boldsymbol{M} \cdot \boldsymbol{U})
$$

For analytical convenience, a projection operator $\boldsymbol{P}_{\alpha}$ in the generalized vector space is introduced and defined as

$$
\boldsymbol{P}_{\alpha}=p_{i j}^{\alpha} \boldsymbol{e}_{i} \boldsymbol{e}_{j} \quad(\alpha \in\{1, \ldots, N\} ; i=1,2 ; j=1, \ldots, N),
$$

where

$$
p_{i j}^{\alpha}= \begin{cases}1 & (j=2(\alpha-1)+i) \\ 0, & (\text { otherwise })\end{cases}
$$

The dot product of $\boldsymbol{P}_{\alpha}$ and (4.2),

$$
-\frac{\mathrm{d}}{\mathrm{d} t} \boldsymbol{P}_{\alpha} \cdot(\boldsymbol{A} \cdot \boldsymbol{U})+\boldsymbol{P}_{\alpha} \cdot \frac{\partial T}{\partial \boldsymbol{X}}=\frac{\mathrm{d}}{\mathrm{d} t} \boldsymbol{P}_{\alpha} \cdot(\boldsymbol{M} \cdot \boldsymbol{U}),
$$

results in the following dynamical equation of motion:

$$
\frac{\mathrm{d}}{\mathrm{d} t} \int_{c_{\alpha}} \rho \phi \boldsymbol{n}_{\alpha} \mathrm{d} l_{\alpha}+\frac{\partial T_{f}}{\partial \boldsymbol{x}_{\alpha}}=m_{\alpha} \frac{\mathrm{d} \boldsymbol{u}_{\alpha}}{\mathrm{d} t} \quad(\alpha \in\{1, \ldots, N\}) .
$$

Considering all singularities spread respectively within individual boundary curves, it is readily proved that

$$
\frac{\partial T_{f}}{\partial \boldsymbol{x}_{\alpha}}=-\frac{\rho}{2} \frac{\partial}{\partial \boldsymbol{x}_{\alpha}} \int_{\Sigma c_{\beta}} \phi \frac{\partial \phi}{\partial n_{\beta}} \mathrm{d} l_{\beta}=-\frac{\rho}{2} \int_{c_{\alpha}}\left(\frac{\partial \phi}{\partial \boldsymbol{x}_{\alpha}} \mathrm{d} \psi-\frac{\partial \psi}{\partial \boldsymbol{x}_{\alpha}} \mathrm{d} \phi\right) \quad(\alpha \in\{1, \ldots, N\}) .
$$

Since singularity $\alpha$ is a function of $\boldsymbol{x}-\boldsymbol{x}_{\alpha}$, it gives $\partial / \partial \boldsymbol{x}_{\alpha}=-\partial / \partial \boldsymbol{x}$. As a result,

$$
\frac{\partial T_{f}}{\partial \boldsymbol{x}_{\alpha}}=\frac{\rho}{2} \int_{c_{\alpha}}\left(\frac{\partial \phi}{\partial \boldsymbol{x}} \mathrm{d} \psi-\frac{\partial \psi}{\partial \boldsymbol{x}} \mathrm{d} \phi\right)=\frac{\rho}{2} \int_{c_{\alpha}}(\nabla \phi \mathrm{d} \psi-\nabla \psi \mathrm{d} \phi)
$$




$$
\begin{aligned}
= & \frac{\rho}{2} \int_{c_{\alpha}}\left(\boldsymbol{e}_{1} \frac{\partial \phi}{\partial x_{1}}+\boldsymbol{e}_{2} \frac{\partial \phi}{\partial x_{2}}\right)\left(\mathrm{d} x_{2} \frac{\partial \phi}{\partial x_{1}}-\mathrm{d} x_{1} \frac{\partial \phi}{\partial x_{2}}\right) \\
& -\frac{\rho}{2} \int_{c_{\alpha}}\left(-\boldsymbol{e}_{1} \frac{\partial \phi}{\partial x_{2}}+\boldsymbol{e}_{2} \frac{\partial \phi}{\partial x_{1}}\right)\left(\mathrm{d} x_{1} \frac{\partial \phi}{\partial x_{1}}+\mathrm{d} x_{2} \frac{\partial \phi}{\partial x_{2}}\right) \\
= & \frac{\rho}{2} \int_{c_{\alpha}}\left(\boldsymbol{e}_{1} \frac{\mathrm{d} x_{2}}{\mathrm{~d} l_{\alpha}} \nabla \phi \cdot \nabla \phi-\boldsymbol{e}_{2} \frac{\mathrm{d} x_{1}}{\mathrm{~d} l_{\alpha}} \nabla \phi \cdot \nabla \phi\right) \mathrm{d} l_{\alpha} \\
= & \frac{\rho}{2} \int_{c_{\alpha}} \boldsymbol{n}_{\alpha} \nabla \phi \cdot \nabla \phi \mathrm{d} l_{\alpha} \quad(\alpha \in\{1, \ldots, N\}) .
\end{aligned}
$$

Equations (4.6) and (4.8) tell us that the left-hand side of (4.6) represents the same force acting on circle $\alpha$ as $(4.1 b)$. Therefore, the derivation reveals that the energy-based Lagrangian framework is equivalent in dynamics to momentum-type one. The equivalence implies that the pressure integration around a submerged body is holographic, which provides information about accelerations of all bodies. These accelerations are coupled with one another by added masses, and none of the others can be determined if some acceleration is unknown.

\section{Dynamical analyses}

Equations (3.12), (3.7), (3.8b), (A 12b) and (A 13b) are applied to determine the hydrodynamic interaction among $N$ circular cylinders planar-translating in an inviscid liquid and to predict the velocity and position of each cylinder at any time. As their analytical solution is generally not available, the numerical calculation is a vital way of solving these algebraic and nonlinear ordinary differential equations with given initial values. There exist two sources of error which influence the prediction of dynamical behaviours of the moving cylinders, one resulting from the numerical solution of ordinary differential equations (3.12) and the other from truncating the series. To present an accurate prediction, (3.12) is solved using the fourth-order Runge-Kutta-Fehlberg method of integration, and the time step is adaptive according to an error tolerance of five significant figures. In the description of translations of the circular cylinders, as the gap between any two cylinders is narrowed down to a relative interval of $5 \times 10^{-4}$ to the smaller one, numerical calculation will automatically stop although they cannot come into contact at all in theory. Since each of the $i$ th terms in the series expressions in (3.7) behaves like $\max \left\{\hat{s}_{\alpha \beta}^{-2 i} \mid \alpha, \beta=1, \ldots, N ; \alpha \neq \beta\right\}$, where $\hat{s}_{\alpha \beta}$ is the centre-to-centre distance between cylinders $\alpha$ and $\beta$, the truncated series in an extreme case at $i=60$ would make our numerical results accurate up to the 120th inverse power of $\hat{s}_{\alpha \beta}$ to meet the accuracy requirement of the current calculation examples. The present calculations are carried out with an automatic increase in $i$ by 20 until the summation of these terms produces no significant variation within the preassigned level of accuracy. Obviously, with an increase in the number of submerged cylinders, the computing time increases rapidly. A rough estimate is made that the prediction of $N$ moving circular cylinders involves calculations of $N^{2} \times N_{0}$ offset functions, where $N_{0}$ denotes the total cyclic number, and $3 N(2 N+1)$ coefficients of the added-mass matrix and its derivatives. This means that the computing time is of $O\left(N^{2}\right)$. In what follows, each physical variable is expressed by means of the 
corresponding dimensionless variable with superscript $*$, and $L_{c}, U_{c}, L_{c} / U_{c}$, and $\rho$ are selected as the characteristic length, velocity, time, and density respectively.

\subsection{Hydrodynamic interactions between two cylinders}

Translations of two submerged circular cylinders $(N=2)$ are predicted and the results are compared with those available in the literature. In this case, expressions for added-mass coefficients can be written as

$$
\left.\begin{array}{l}
A_{11}+\mathrm{i} A_{12}=2 \pi \rho R_{1}^{2}\left[0.5+\sum_{i=1}^{\infty} \lambda_{1111}^{(2 i)}\right], \\
A_{21}+\mathrm{i} A_{22}=2 \pi \rho R_{1}^{2} \mathrm{i}\left[0.5+\sum_{i=1}^{\infty} \lambda_{1211}^{(2 i)}\right], \\
A_{31}+\mathrm{i} A_{32}=2 \pi \rho R_{2}^{2} \sum_{i=0}^{\infty} \lambda_{2111}^{(2 i+1)}, \\
A_{41}+\mathrm{i} A_{42}=2 \pi \rho R_{2}^{2} \mathrm{i} \sum_{i=0}^{\infty} \lambda_{2211}^{(2 i+1)}, \\
A_{33}+\mathrm{i} A_{34}=2 \pi \rho R_{2}^{2}\left[0.5+\sum_{i=1}^{\infty} \lambda_{2111}^{(2 i)}\right], \\
A_{43}+\mathrm{i} A_{44}=2 \pi \rho R_{2}^{2} \mathrm{i}\left[0.5+\sum_{i=1}^{\infty} \lambda_{2211}^{(2 i)}\right],
\end{array}\right\}
$$

where recurrence formulae of $\lambda_{\alpha j m 1}^{(i)}$ are

$$
\left.\begin{array}{l}
\lambda_{1 j m 1}^{(0)}=0, \quad \lambda_{1 j m 1}^{(1)}=(-1)^{m+j-1} H\left(m, 1, R_{2}, \bar{s}_{12}\right), \\
\lambda_{1 j m 1}^{(2 i)}=\sum_{l=1}^{\infty}(-1)^{l+j-1} H\left(m, l, R_{1}, \bar{s}_{12}\right) \bar{\lambda}_{1 j l 1}^{(2 i-1)}, \\
\lambda_{1 j m 1}^{(2 i+1)}=(-1)^{m+j-1} \sum_{l=1}^{\infty} H\left(m, l, R_{2}, \bar{s}_{12}\right) \bar{\lambda}_{1 j l 1}^{(2 i)},
\end{array}\right\}
$$

and

$$
\left.\begin{array}{l}
\lambda_{2 j m 1}^{(0)}=0, \quad \lambda_{2 j m 1}^{(1)}=(-1)^{j} H\left(m, 1, R_{1}, \bar{s}_{12}\right), \\
\lambda_{2 j m 1}^{(2 i)}=(-1)^{m+j-1} \sum_{l=1}^{\infty} H\left(m, l, R_{2}, \bar{s}_{12}\right) \bar{\lambda}_{2 j l 1}^{(2 i-1)}, \\
\lambda_{2 j m 1}^{(2 i+1)}=\sum_{l=1}^{\infty}(-1)^{l+j-1} H\left(m, l, R_{1}, \bar{s}_{12}\right) \bar{\lambda}_{2 j l 1}^{(2 i)} \quad(j=1,2 ; i=1, \ldots, \infty) .
\end{array}\right\}
$$

Equations (3.12), (3.8b), (5.1a), (5.1b) and (5.1c) are employed to predict the dynamical behaviour of two submerged moving circular cylinders. When the two bodies are not too far apart and there are weak hydrodynamic interactions between 
them, the added-mass coefficients can be simplified to the order of $O\left(\left|s_{12}\right|^{-2}\right)$, i.e.

$$
\left.\begin{array}{rl}
A_{11}+\mathrm{i} A_{12} & \approx \pi \rho R_{1}^{2}, \\
A_{21}+\mathrm{i} A_{22} & \approx \pi \rho R_{1}^{2} \mathrm{i}, \\
A_{31}+\mathrm{i} A_{32} & \approx-2 \pi \rho R_{2}^{2} R_{1}^{2} / \bar{s}_{12}^{2}, \\
A_{41}+\mathrm{i} A_{42} & \approx 2 \pi \rho R_{2}^{2} R_{1}^{2} \mathrm{i} / \bar{s}_{12}^{2}, \\
A_{33}+\mathrm{i} A_{34} & \approx \pi \rho R_{2}^{2}, \\
A_{43}+\mathrm{i} A_{44} & \approx \pi \rho R_{2}^{2} \mathrm{i} .
\end{array}\right\}
$$

Let us first consider a drifting circular cylinder $(\alpha=1)$ around another fixed one $(\alpha=2)$. The uniform flow is $U_{0}=1$ along the $x$-direction at infinity. The initial position and velocity of the moving cylinder of radius $R_{1}^{*}=0.1$ are $x_{110}^{*}=-20$ and $\boldsymbol{u}_{10}^{*}=(1,0)$ where $L_{c}=R_{2}=1$ and $U_{c}=U_{0}$. Figure 2 shows trajectories of drifting cylinder 1 with different initial transverse positions. In the figure, in addition to results from the current formulation, data given by Guo \& Chwang (1991) are also plotted for comparison. It is obvious that our results agree well with what they predicted. Figure 3 exhibits a free-free interaction between two circular cylinders and the result is compared to that of Tchieu et al. (2010) with assumptions $R_{\alpha}=L_{c}=1$ and $\rho=\rho_{\alpha} \quad(\alpha=1,2)$. It is noted that the current approach predicts the same phenomenon as Tchieu et al. did that the incoming cylinder deviates downward from a set course without a direct contact with the initially stationary one, and the latter gains a substantial amount of momentum to move upward. However, there are slight differences between the two results in the trajectory and terminal velocity. The current method predicts two terminal velocities of $\boldsymbol{u}_{1 \infty}^{*}=(0.943,-0.230)$ and $\boldsymbol{u}_{2 \infty}^{*}=(0.044,0.236)$, while those from Tchieu et al. are $\boldsymbol{u}_{1 \infty}^{*}=(0.954,-0.205)$ and $\boldsymbol{u}_{2 \infty}^{*}=(0.030,0.216)$. The discrepancies may result from whether the influence of a non-uniform added-mass distribution on all acceleration terms is taken into account. In the two cases, moreover, every trajectory is obtained numerically in tens of minutes by a laptop. Another example brings out considerations on the relativity of the moving bodies. Let us consider two identical circular cylinders translating along the $x$-axis. Two cases are given with distinct initial kinematic conditions in which the initial velocities of the bodies are distinct but their velocity differences remain the same, as seen in figure 4 . Note from figure $4(a)$ that when the two cylinders move at the same initial speed but in opposite directions, their velocity-time curves are symmetric with respect to the line $u^{*}=0$. This means that equal hydrodynamic forces are exerted on each of them. But note from figure $4(b)$ that if the two cylinders translate one after the other in the same direction, their velocity-time curves appear asymmetric, although for an observer in an inertial reference frame with a relative velocity $u_{0}=1.25$, the initial velocities of the two cylinders remain unchanged. This indicates that different hydrodynamic forces act on each cylinder. The two cases remind us that Galilean relativity seems to be unsuitable for describing the moving bodies under hydrodynamic interaction. An explanation for the phenomenon follows.

As the two cylinders are translating along the $x$-axis, $\bar{s}_{12}=s=x_{21}-x_{11}, \partial / \partial x_{11}=$ $-\mathrm{d} / \mathrm{d} s, \partial / \partial x_{21}=\mathrm{d} / \mathrm{d} s, \mathrm{~d} / \mathrm{d} t=\left(u_{21}-u_{11}\right) \mathrm{d} / \mathrm{d} s$, and all $\lambda$ are real numbers. Therefore, the coefficient matrix $\left[A_{i, j}\right]$ of instantaneous added-mass tensor $\boldsymbol{A}$ and the generalized 


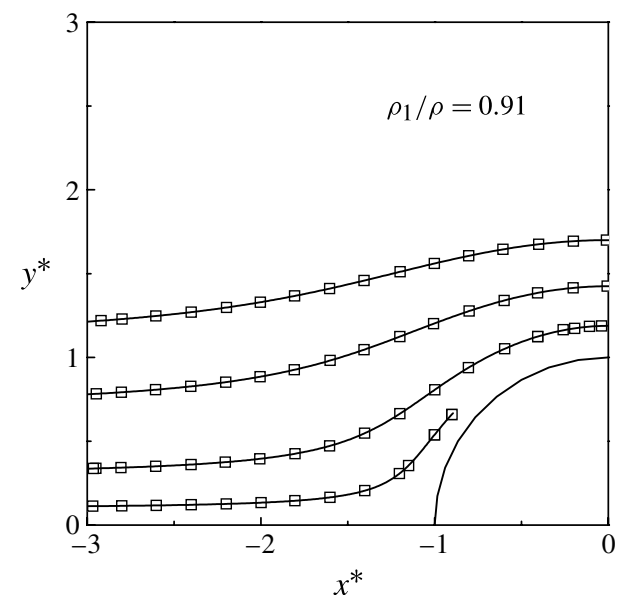

FIGURE 2. Trajectories of a drifting cylinder with various initial transverse positions, $\boldsymbol{x}_{120}^{*}=0.1,0.3,0.7$ and 1.1. Present method (solid lines); data from Guo \& Chwang (1991) (open squares).

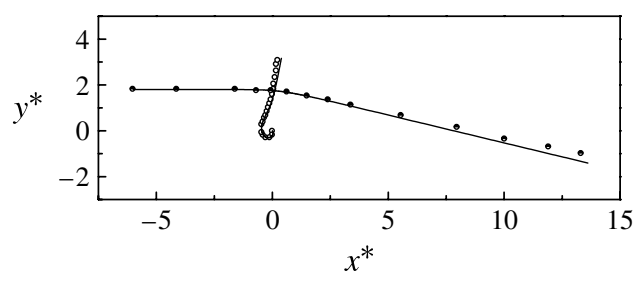

FIgURE 3. Cylinder trajectories for a free-free interaction. The initial conditions are $\boldsymbol{x}_{10}^{*}=(-6,1.8), \boldsymbol{x}_{20}^{*}=(0,0), \boldsymbol{u}_{10}^{*}=(1,0)$, and $\boldsymbol{u}_{20}^{*}=(0,0)$. Present method (solid lines); data from Tchieu et al. (2010) (circles).
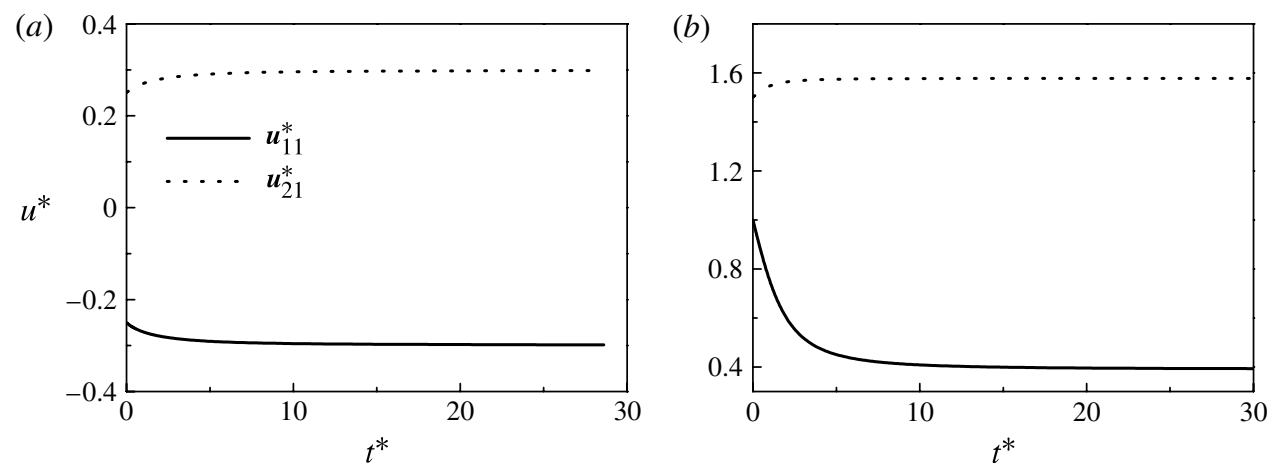

FIGURE 4. Variation of translational velocities of two circular cylinders against time with $\rho_{i}^{*}=0.5, R_{i}^{*}=1.0(i=1,2)$. The initial positions are $\boldsymbol{x}_{10}^{*}=(0,0), \boldsymbol{x}_{20}^{*}=(2.2,0)$ and the initial velocities are $(a) \boldsymbol{u}_{10}^{*}=(-0.25,0), \boldsymbol{u}_{20}^{*}=(0.25,0)$ and $(b) \boldsymbol{u}_{10}^{*}=(1.0,0)$, $\boldsymbol{u}_{20}^{*}=(1.5,0)$. 
velocity vector become

$$
\left[A_{i, j}\right]=\left[\begin{array}{cccc}
A_{11} & 0 & A_{31} & 0 \\
0 & A_{22} & 0 & A_{42} \\
A_{31} & 0 & A_{33} & 0 \\
0 & A_{42} & 0 & A_{44}
\end{array}\right] \quad \text { and } \quad\left[U_{i}\right]=\left[\begin{array}{c}
u_{11} \\
0 \\
u_{21} \\
0
\end{array}\right]
$$

Based on recurrence formulae $(5.1 b)$ and $(5.1 c)$, it is easy to find that $\lambda_{\alpha 1 m 1}^{(k)}=$ $(-1)^{k} \lambda_{\alpha 2 m 1}^{(k)}(k=1,2, \ldots)$, which leads to $A_{11}=A_{22}, A_{33}=A_{44}$ and $A_{31}=-A_{42}$.

Substituting the above-mentioned $\boldsymbol{A}$ and $\boldsymbol{U}$ into (3.12) results in two dynamical equations of motion,

$$
\left(m_{1}+A_{11}\right) \frac{\mathrm{d} u_{11}}{\mathrm{~d} t}+A_{31} \frac{\mathrm{d} u_{21}}{\mathrm{~d} t}=\frac{\mathrm{d} A_{11}}{\mathrm{~d} s} u_{11}\left(\frac{u_{11}}{2}-u_{21}\right)-u_{21}^{2} \frac{\mathrm{d}}{\mathrm{d} s}\left(A_{31}+\frac{A_{33}}{2}\right)
$$

and

$$
A_{31} \frac{\mathrm{d} u_{11}}{\mathrm{~d} t}+\left(m_{2}+A_{33}\right) \frac{\mathrm{d} u_{21}}{\mathrm{~d} t}=-\frac{\mathrm{d} A_{33}}{\mathrm{~d} s} u_{21}\left(\frac{u_{21}}{2}-u_{11}\right)+u_{11}^{2} \frac{\mathrm{d}}{\mathrm{d} s}\left(A_{31}+\frac{A_{11}}{2}\right) .
$$

Seen from an inertial reference frame with a relative velocity $u_{0}$, velocities of the two cylinders are $u_{11}^{\prime}$ and $u_{21}^{\prime}$, respectively, i.e. $u_{11}=u_{0}+u_{11}^{\prime}$ and $u_{21}=u_{0}+u_{21}^{\prime}$. Putting these two expressions into (5.5) leads to

$$
\begin{aligned}
\left(m_{1}+A_{11}\right) \frac{\mathrm{d} u_{11}^{\prime}}{\mathrm{d} t}+A_{31} \frac{\mathrm{d} u_{21}^{\prime}}{\mathrm{d} t}= & \frac{\mathrm{d} A_{11}}{\mathrm{~d} s} u_{11}^{\prime}\left(\frac{u_{11}^{\prime}}{2}-u_{21}^{\prime}\right)-u_{21}^{\prime 2} \frac{\mathrm{d}}{\mathrm{d} s}\left(A_{31}+\frac{A_{33}}{2}\right) \\
& -u_{0}\left(\frac{u_{0}}{2}+u_{21}^{\prime}\right) \frac{\mathrm{d}}{\mathrm{d} s}\left(A_{11}+A_{33}+2 A_{31}\right)
\end{aligned}
$$

and

$$
\begin{aligned}
A_{31} \frac{\mathrm{d} u_{11}^{\prime}}{\mathrm{d} t}+\left(m_{2}+A_{33}\right) \frac{\mathrm{d} u_{21}^{\prime}}{\mathrm{d} t}= & -\frac{\mathrm{d} A_{33}}{\mathrm{~d} s} u_{21}^{\prime}\left(\frac{u_{21}^{\prime}}{2}-u_{11}^{\prime}\right)+u_{11}^{\prime 2} \frac{\mathrm{d}}{\mathrm{d} s}\left(A_{31}+\frac{A_{11}}{2}\right) \\
& +u_{0}\left(\frac{u_{0}}{2}+u_{11}^{\prime}\right) \frac{\mathrm{d}}{\mathrm{d} s}\left(A_{11}+A_{33}+2 A_{31}\right) .
\end{aligned}
$$

Therefore, an observer in that frame of reference should add two corrective hydrodynamic forces to (5.5) so as to describe the same dynamical behaviour of the two moving cylinders.

\subsection{Dynamical behaviours of the three translating circular cylinders}

In this section, let us discuss planar translations of three submerged circular cylinders in order to expand our horizon regarding the nonlinear behaviour of many bodies moving through a liquid. As $N=3$, the number of independent instantaneous addedmass coefficients is 21 , and (3.7) may be reduced to

$$
\begin{aligned}
& A_{2(\alpha-1)+j, 2 \gamma-1}+\mathrm{i} A_{2(\alpha-1)+j, 2 \gamma}=2 \pi \rho R_{\alpha}^{2} \mathrm{i}^{j-1}\left[0.5 \delta_{\alpha \gamma}+\sum_{i=0}^{\infty} \lambda_{\alpha j 11}^{(3 i+(3+\gamma-\alpha) \bmod 3)}\right] \\
& (\alpha, \gamma \in\{1,2,3\} ; j=1 \text { or } 2),
\end{aligned}
$$


where recurrence formulae of $\lambda_{\alpha j 11}^{(i)}$ are

$$
\left.\begin{array}{l}
\lambda_{\alpha j 11}^{(0)}=0, \quad \lambda_{\alpha j 11}^{(1)}=(-1)^{j} H\left(1,1, R_{(\alpha+1)^{\dagger}}, \bar{s}_{\alpha,(\alpha+1)^{\dagger}}\right), \\
\lambda_{\alpha j 11}^{(2)}=(-1)^{j}\left[H\left(1,1, R_{(\alpha+2)^{\dagger}}, \bar{s}_{\alpha,(\alpha+2)^{\dagger}}\right)+\sum_{l=1}^{\infty} H\left(1, l, R_{(\alpha+2)^{\dagger}}, \bar{s}_{\left.(\alpha+1)^{\dagger},(\alpha+2)^{\dagger}\right)} \bar{\lambda}_{\alpha j l 1}^{(1)}\right],\right. \\
\lambda_{\alpha j 11}^{(3 i)}=(-1)^{j} \sum_{l=1}^{\infty} \sum_{\gamma=1}^{2} H\left(1, l, R_{\alpha}, \bar{s}_{(\alpha+\gamma)^{\dagger}, \alpha}\right) \bar{\lambda}_{\alpha j l 1}^{(3 i-3+\gamma)}, \\
\lambda_{\alpha j 11}^{(3 i+1)}=(-1)^{j} \sum_{l=1}^{\infty} \sum_{\gamma=1}^{2} H\left(1, l, R_{(\alpha+1)^{\dagger}}, \bar{s}_{(\alpha+\gamma+1)^{\dagger},(\alpha+1)^{\dagger}}\right) \bar{\lambda}_{\alpha j l 1}^{(3 i-2+\gamma)}, \\
\lambda_{\alpha j 11}^{(3 i+2)}=(-1)^{j} \sum_{l=1}^{\infty} \sum_{\gamma=1}^{2} H\left(1, l, R_{(\alpha+2)^{\dagger}}, \bar{s}_{(\alpha+\gamma-1)^{\dagger},(\alpha+2)^{\dagger}}\right) \bar{\lambda}_{\alpha j l 1}^{(3 i-1+\gamma)} \quad(i=1, \ldots, \infty) .
\end{array}\right\}
$$

When the three cylinders are not too far apart and there are weak hydrodynamic interactions between them, the added-mass coefficients can be simplified to the order of $O\left(\left|s_{12}\right|^{-i}\left|s_{23}\right|^{-j}\left|s_{31}\right|^{-k}\right)(i+j+k=2)$, i.e.

$$
\begin{aligned}
& A_{11}+\mathrm{i} A_{12} \approx \pi \rho R_{1}^{2}, \\
& A_{21}+\mathrm{i} A_{22} \approx \pi \rho R_{1}^{2} \mathrm{i}, \\
& A_{31}+\mathrm{i} A_{32} \approx-2 \pi \rho R_{2}^{2} R_{1}^{2} / \bar{s}_{12}^{2}, \\
& A_{41}+\mathrm{i} A_{42} \approx 2 \pi \rho R_{2}^{2} R_{1}^{2} \mathrm{i} / \bar{s}_{12}^{2}, \\
& A_{51}+\mathrm{i} A_{52} \approx-2 \pi \rho R_{3}^{2} R_{1}^{2} / \bar{s}_{31}^{2}, \\
& A_{61}+\mathrm{i} A_{62} \approx 2 \pi \rho R_{3}^{2} R_{1}^{2} \mathrm{i} / \bar{s}_{31}^{2}, \\
& A_{33}+\mathrm{i} A_{34} \approx \pi \rho R_{2}^{2}, \\
& A_{43}+\mathrm{i} A_{44} \approx \pi \rho R_{2}^{2} \mathrm{i}, \\
& A_{53}+\mathrm{i} A_{54} \approx-2 \pi \rho R_{3}^{2} R_{2}^{2} / \bar{s}_{23}^{2}, \\
& A_{63}+\mathrm{i} A_{64} \approx 2 \pi \rho R_{3}^{2} R_{2}^{2} \mathrm{i} / \bar{s}_{23}^{2}, \\
& A_{55}+\mathrm{i} A_{56} \approx \pi \rho R_{3}^{2}, \\
& A_{65}+\mathrm{i} A_{66} \approx \pi \rho R_{3}^{2} \mathrm{i} .
\end{aligned}
$$

Based on (3.12), (3.8b), (5.8a) and (5.8b), the dynamical behaviour of three planartranslating circular cylinders under fully hydrodynamic interactions can be predicted numerically. The following are some interesting results.

When a large body translates initially at a higher speed than that of a trailing smaller one, what will happen? Traditionally, the large body would move farther and farther away from the following one. However, in a fully coupled three-body system, the moving pattern is quite different. Figure $5(a)$ shows that for given parameters and initial conditions, one trailing smaller circular cylinder translates initially at a lower speed than those of two leading large ones, but after a short period of time it will overtake the two forerunners. Variations of their velocities in the same time interval are plotted in figure $5(b)$, which presents an illustration for the phenomenon. 

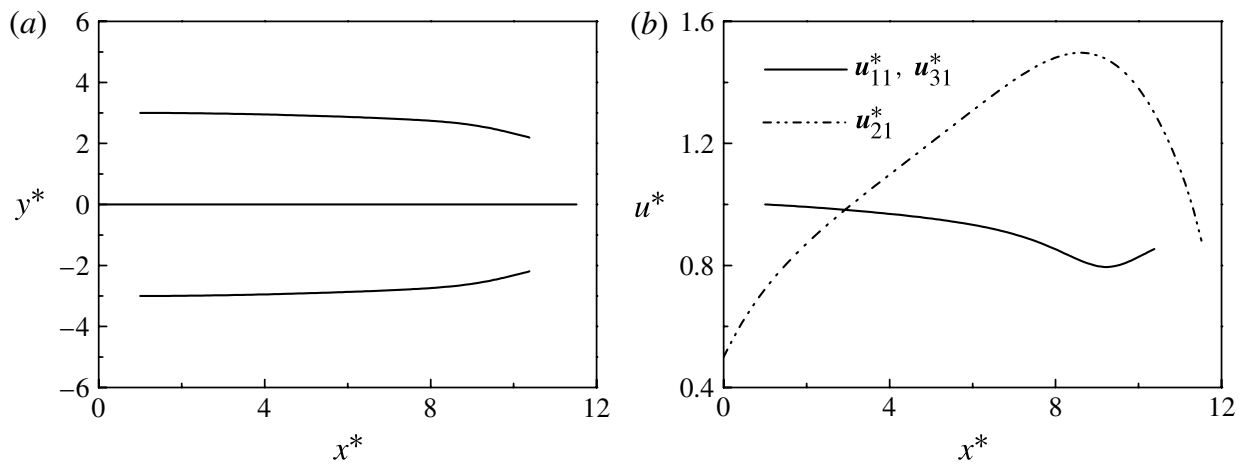

Figure 5. (a) Cylinder trajectories for a free-free interaction with $\rho_{i}^{*}=0.8(i=1,2,3)$, $R_{1}^{*}=R_{3}^{*}=1$, and $R_{2}^{*}=0.5 ;(b)$ variations of their velocities in the $x$-direction. The initial conditions are $\boldsymbol{x}_{10}^{*}=(1,3), \boldsymbol{x}_{20}^{*}=(0,0), \boldsymbol{x}_{30}^{*}=(1,-3), \boldsymbol{u}_{10}^{*}=\boldsymbol{u}_{30}^{*}=(1,0)$ and $\boldsymbol{u}_{20}^{*}=(0.5,0)$.
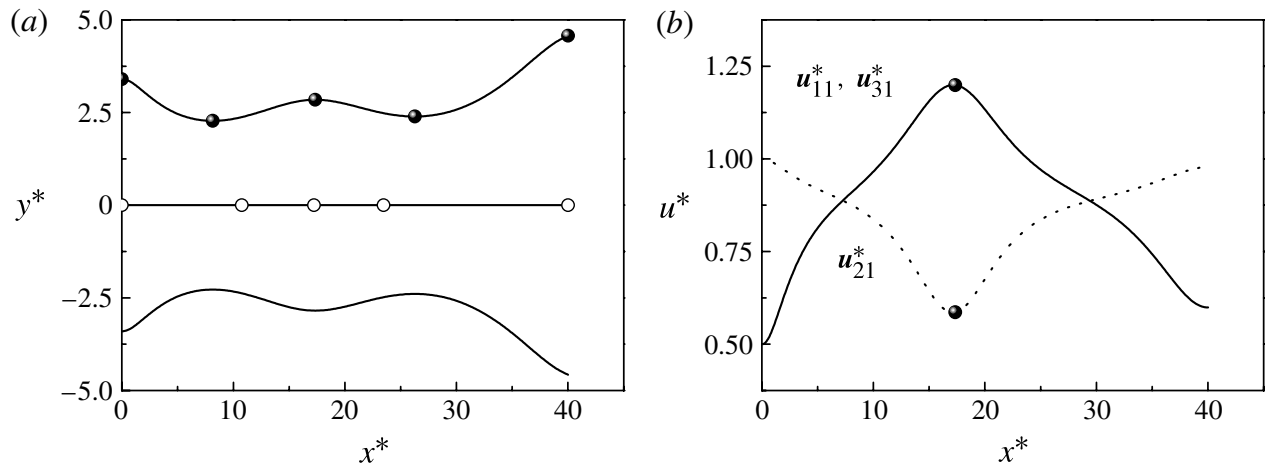

Figure 6. (a) Cylinder trajectories for a free-free interaction with $\rho_{i}^{*}=0.8(i=1,2,3)$, $R_{1}^{*}=R_{3}^{*}=0.5$, and $R_{2}^{*}=1$ (white and black circles indicate five specific instants, respectively); $(b)$ variations of their velocities in the $x$-direction (black circles indicate extreme positions). The initial conditions are $\boldsymbol{x}_{10}^{*}=(0,3.4), \boldsymbol{x}_{20}^{*}=(0,0), \boldsymbol{x}_{30}^{*}=(0,-3.4)$, $\boldsymbol{u}_{10}^{*}=\boldsymbol{u}_{30}^{*}=(0.5,0)$ and $\boldsymbol{u}_{20}^{*}=(1,0)$.

It is noticed from the figure that the velocities of two initially fast-translating large cylinders gradually decrease in the $x$-axis direction with their approach to each other; on the contrary, the velocity of the initially slow-translating trailing one rapidly increases, and even greatly exceeds those of the two large ones in a short time. The turning points of these velocities appear when the smaller cylinder just overtakes the large ones in front. Therefore, one would observe an ejection phenomenon such that two initially fast-translating large cylinders pull the initially slow-translating following one moving in the same direction, and then eject it in front of them rather than preventing it from approaching. This means that in the fully coupled system, part of the kinetic energy of the two leading large cylinders is transferred to the trailing smaller one through the medium of the liquid.

Another nonlinear behaviour of three bodies due to the hydrodynamic interaction is illustrated in figure 6. Figure 6(a) shows that when a large circular cylinder is inserted in the middle of two smaller identical ones and set at a higher initial translation 


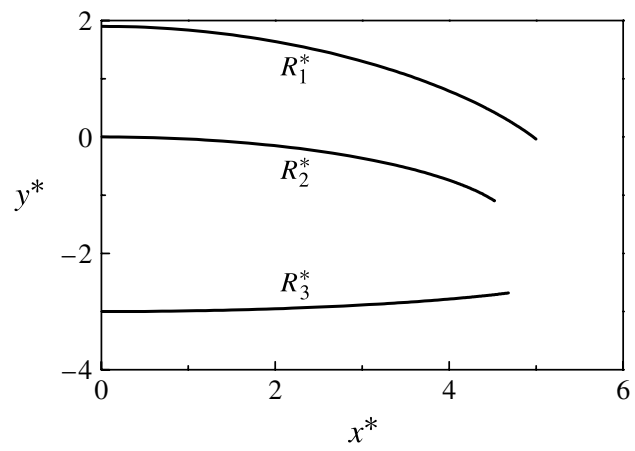

FIGURE 7. Cylinder trajectories for a free-free interaction with $\rho_{i}^{*}=0.8(i=1,2,3)$, $R_{1}^{*}=0.25, R_{2}^{*}=0.5$ and $R_{3}^{*}=1$. The initial conditions are $x_{10}^{*}=(0,1.9), x_{20}^{*}=(0,0)$, $\boldsymbol{x}_{30}^{*}=(0,-3)$, and $\boldsymbol{u}_{i 0}^{*}=(1,0)(i=1,2,3)$.

speed than its two partners, the paths of the two smaller ones are sinuous. And carefully checking several specific instants (see white and black circles) one may observe that the large cylinder and the two smaller ones at its two sides would alternately chase after each other. Figure $6(b)$ reveals variations of their horizontal velocities in the 'mutual pursuing' process. Note that at the beginning, the horizontal velocity of the large cylinder continuously decreases down to a minimum, and then experiences a reverse velocity change, almost forming a symmetrical curve with respect to $x^{*}=17.34$, whereas the horizontal velocities of the two outer smaller ones increase rapidly up to a maximum at an early stage, and decrease quickly at a later stage. There seems to be an approximate symmetry between the two curves with respect to $u^{*}=0.875$. This illustrates that in the 'mutual pursuing' process the two smaller circular cylinders translate in an oscillatory and velocity-varying manner, and absorb kinetic energy from or give it to the large one.

Figure 7 shows a case such that when three circular cylinders initially side by side translate forward and the two smaller cylinders are both on one side of the large one, the large cylinder will collect the two smaller ones together unless they move too fast to be stopped quickly, which exhibits an aggregating motion.

The behaviour of three identical circular cylinders translating along the $x$-axis is plotted in figure $8(a)$. It is seen that when the cylinders are released at the same initial velocity of unity, the leading cylinder accelerates forwards, whereas the two following ones decelerate, the last one having maximum deceleration. It is obvious that the three velocity curves are asymmetrical with respect to $u^{*}=1$. By comparison of the instantaneous velocities of the three cylinders in figure $8(b)$, one may easily find that the sum of their momentums is not constant although these identical bodies start initially in a uniformly spaced alignment and with the same velocity. Projecting pertinent physical variables in (3.12) onto the $x$-axis, the dynamical equation of motion in this case becomes

$$
\frac{\mathrm{d}}{\mathrm{d} t}\left(A_{2 \alpha-1,1}+A_{2 \alpha-1,3}+A_{2 \alpha-1,5}+m_{\alpha}\right) u_{\alpha 1}=0 \quad(\alpha=1,2,3) .
$$

It stands to reason that the momentum of the liquid should be involved in the motions of these bodies. This reveals that their total momentum is a constant, and accelerations of all the bodies are mutually coupled. 

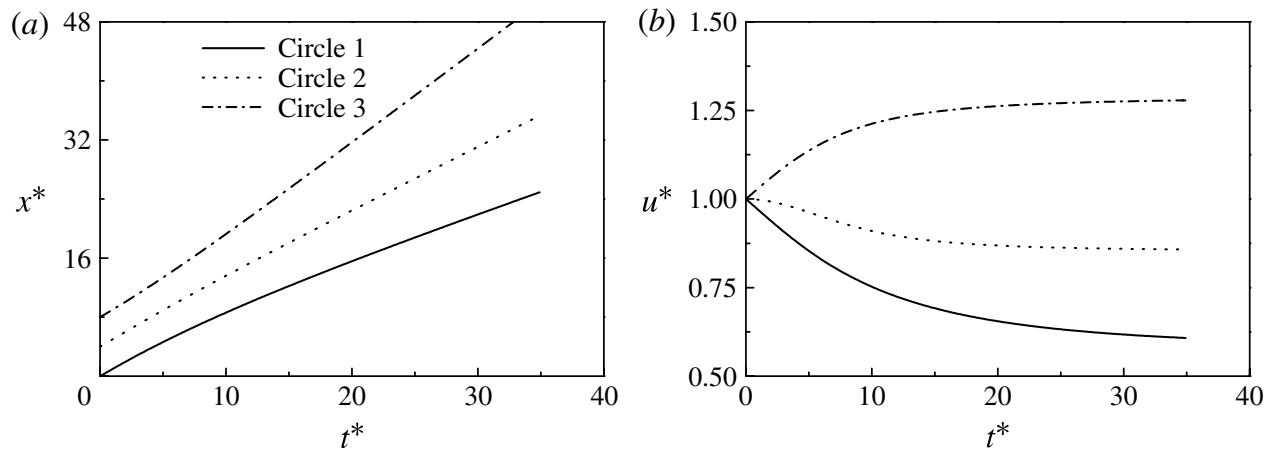

FIgURE 8. (a) Axial displacements of three identical circular cylinders with $\rho_{i}^{*}=0.8$ and $R_{i}^{*}=1(i=1,2,3) ;(b)$ evolution of their velocities. The initial conditions are $\boldsymbol{x}_{10}^{*}=(0,0)$, $\boldsymbol{x}_{20}^{*}=(4,0), \boldsymbol{x}_{30}^{*}=(8,0)$, and $\boldsymbol{u}_{i 0}^{*}=(1,0)(i=1,2,3)$.
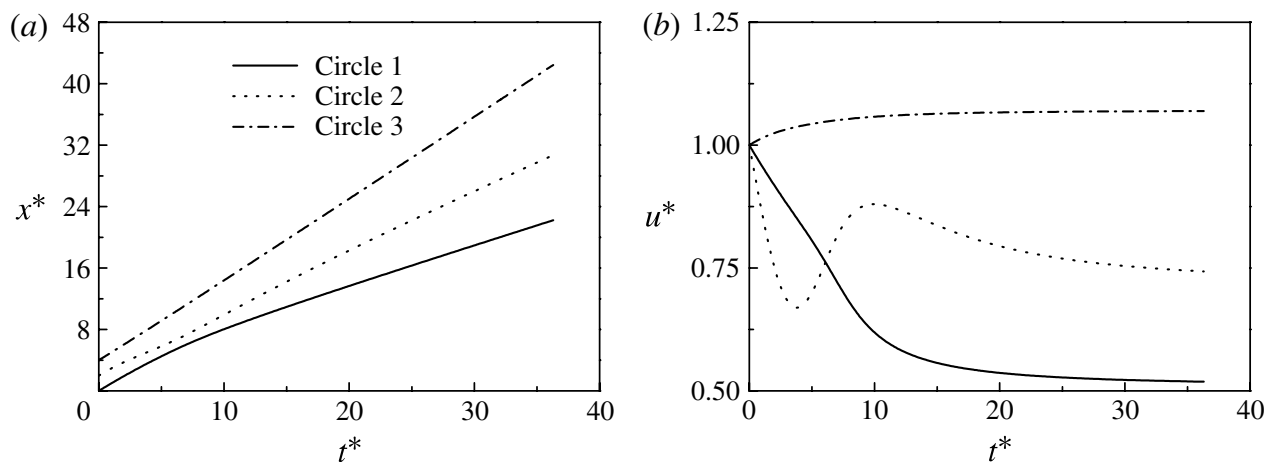

FIgURE 9. (a) Axial displacements of three different-sized circular cylinders with $\rho_{i}^{*}=$ $0.8(i=1,2,3), R_{1}^{*}=0.5, R_{2}^{*}=0.25$, and $R_{3}^{*}=1 ;(b)$ evolution of their velocities. The initial conditions are $\boldsymbol{x}_{10}^{*}=(0,0), \boldsymbol{x}_{20}^{*}=(2,0), \boldsymbol{x}_{30}^{*}=(4,0)$ and $\boldsymbol{u}_{i 0}^{*}=(1,0)(i=1,2,3)$.

Figures 9 and 10 demonstrate other nonlinear translations along the $x$-axis of three different-sized circular cylinders. By comparing figures 9 and 10 with 8, one can observe clearly that in the last two cases, variations of the position and velocity of the largest cylinder with respect to time are much smaller than those of the identical cylinders in figure 8 in spite of smaller initial separation distances between cylinders. This is due to the weaker hydrodynamic interaction the two smaller cylinders induce. However, influence of the largest cylinder on the middle-sized one is still significant although separated by a body, which results in relatively greater variations of the latter position and velocity with respect to time. Regarding the motion of the smallest cylinder, a strong nonlinear phenomenon is discovered. It is noticed from figures $9(b)$ and $10(b)$ that its velocity change is rather rapid and significant during each chase process, appearing wavy in shape. This indicates that at each early stage of the chase the smallest cylinder, under the significant hydrodynamic interaction induced by the largest one, approaches gradually the middle-sized one, but when the hydrodynamic interaction caused by the middle-sized cylinder becomes more powerful than that by the largest one, a resultant force would push it away from the middle-sized 

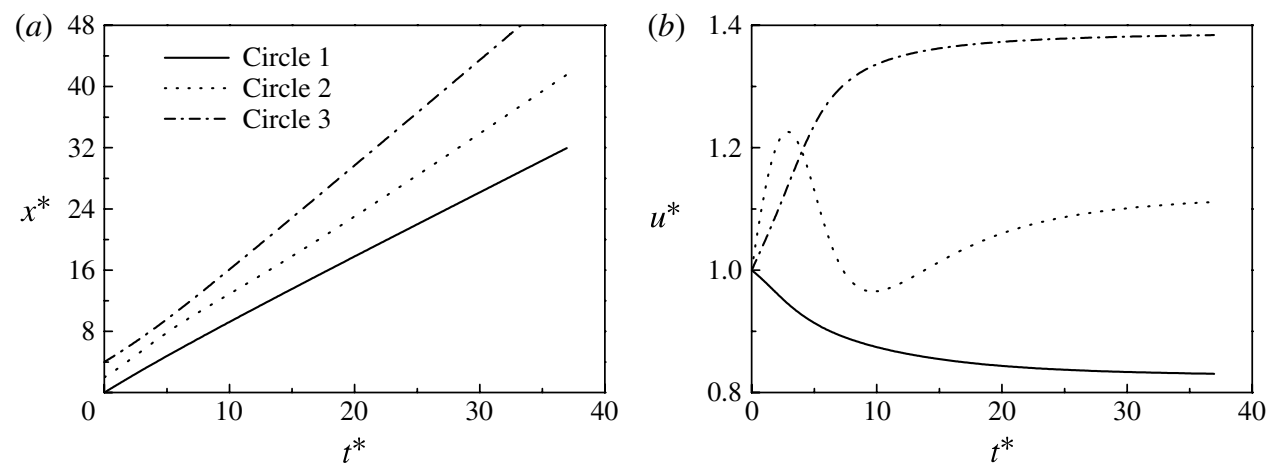

FIGURE 10. (a) Axial displacements of three different-sized circular cylinders with $\rho_{i}^{*}=$ $0.8(i=1,2,3), R_{1}^{*}=1, R_{2}^{*}=0.25$, and $R_{3}^{*}=0.5 ;(b)$ evolution of their velocities. The initial conditions are $\boldsymbol{x}_{10}^{*}=(0,0), \boldsymbol{x}_{20}^{*}=(2,0), \boldsymbol{x}_{30}^{*}=(4,0)$, and $\boldsymbol{u}_{i 0}^{*}=(1,0)(i=1,2,3)$.

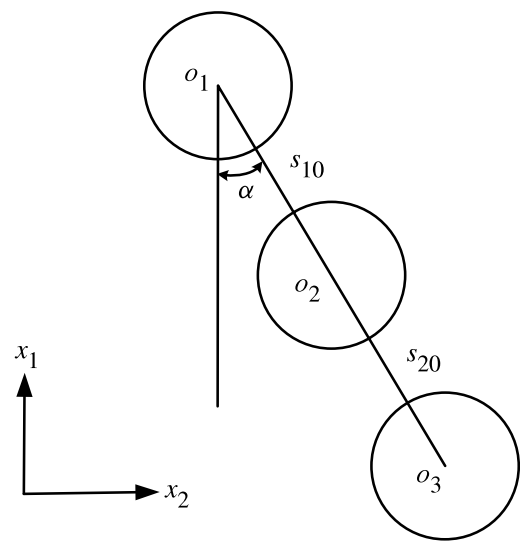

FIGURE 11. Another initial configuration notation for three translating circular cylinders.

cylinder until the influence of the largest one again becomes greater. The nonlinear movement of the smallest cylinder becomes weaker and weaker with a decreasing of the hydrodynamic interaction.

In order to discuss in the following the dynamical behaviours of three translational cylinders conveniently, the initial separation distances $s_{i 0}(i=1,2)$ and angle $\alpha$ of inclination are adopted, as shown in figure 11.

Figure 12 exhibits the complicated nonlinear behaviour of three identical circular cylinders with different initial positions. It is noticed from the figure that with a small initial angle $\alpha$ of inclination, trajectories of the three cylinders appear to be convergent. As $\alpha$ exceeds a specific angle $\alpha_{c}\left(\alpha_{c}=45^{\circ}\right)$, trajectories of the two outer cylinders become divergent. With the initial inclination angle over $75^{\circ}$, the middle cylinder changes its target for pursuit from the one initially behind to the other in front. Two other cases with different-sized cylinders are plotted in figures 13 and 14. It is also seen from these two figures that trajectories of the three cylinders are still convergent but toward the larger one within a range of the initial inclination angle, and as $\alpha$ exceeds $45^{\circ}$, trajectories of the two outer cylinders start to diverge. 

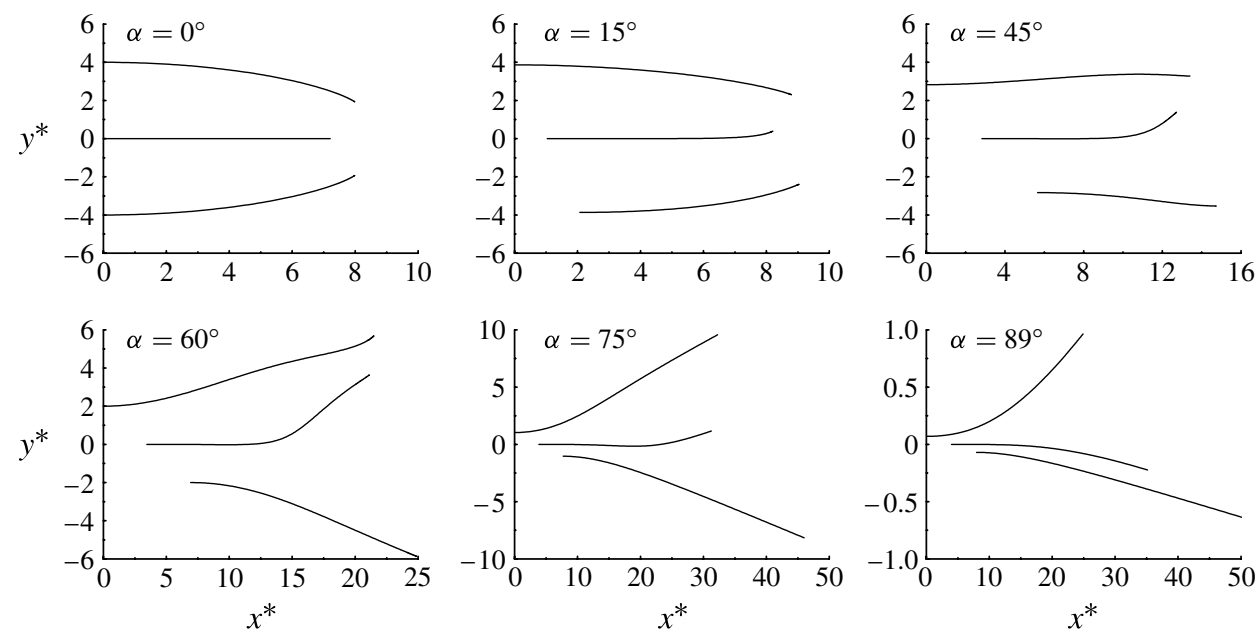

FIGURE 12. Trajectories of three identical circular cylinders of $\rho_{i}^{*}=0.8$ and $R_{i}^{*}=1 \quad(i=$ $1,2,3)$ with initial separation distances, $s_{i 0}^{*}=4(i=1,2)$, and initial translational velocities, $\boldsymbol{u}_{i 0}^{*}=(1,0)(i=1,2,3)$ for various angles $\alpha$.
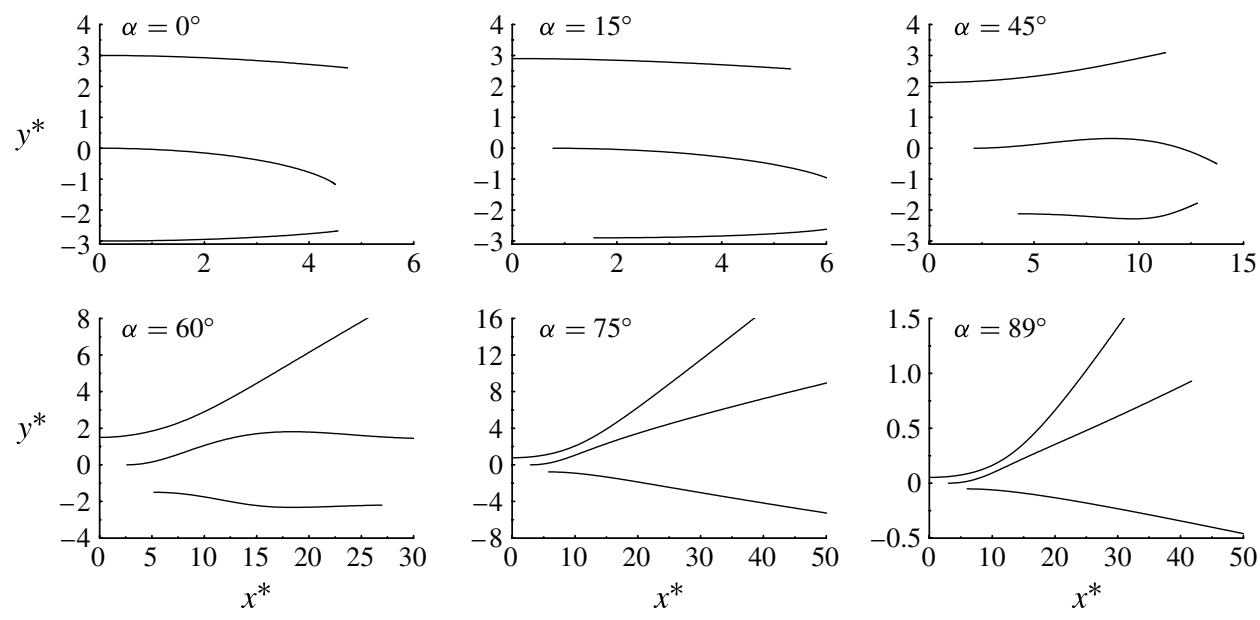

FIGURE 13. Trajectories of three different-sized circular cylinders of $\rho_{i}^{*}=0.8(i=1,2,3)$, $R_{1}^{*}=R_{2}^{*}=0.5$ and $R_{3}^{*}=1$ with initial separation distances, $s_{i 0}^{*}=3(i=1,2)$, and initial translational velocities, $\boldsymbol{u}_{i 0}^{*}=(1,0)(i=1,2,3)$ for various angles $\alpha$.

In figure 13, the middle cylinder is always approaching the large one for an initial condition of $\alpha<75^{\circ}$ due to the aggregating effect, and beyond this critical initial angle it is pushed away from the large one due to an intense repulsive hydrodynamic interaction induced by the large body, whereas in figure 14, the critical value of the initial inclination angle is about $\alpha=60^{\circ}$. Moreover, it is observed from the curve for $\alpha=60^{\circ}$ that the trajectories of the two small cylinders intersect twice during their evolution. One reasonable explanation is that the smallest cylinder first crosses the path of the middle-sized one due to strong repulsive hydrodynamic force induced by the largest one, and with its approach to the middle-sized cylinder, the hydrodynamic 

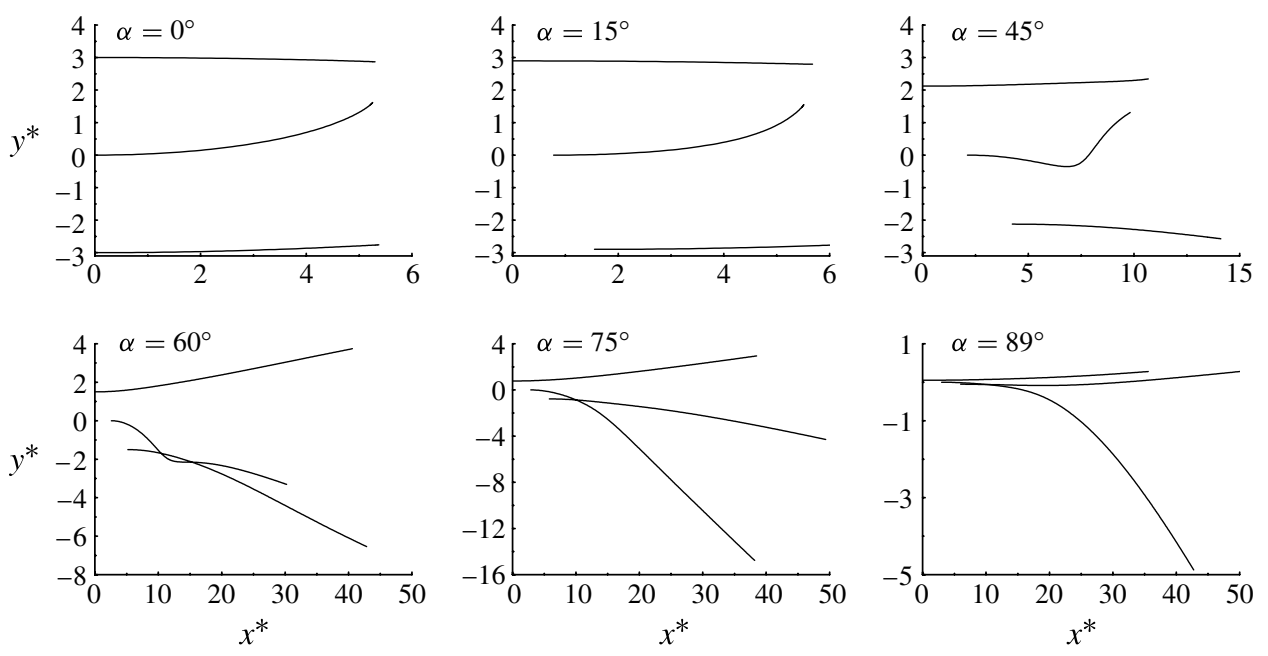

FIGURE 14. Trajectories of three different-sized circular cylinders of $\rho_{i}^{*}=0.8(i=1,2,3)$, $R_{1}^{*}=1, R_{2}^{*}=0.25$ and $R_{3}^{*}=0.5$ with initial separation distances, $s_{i 0}^{*}=3(i=1,2)$, and initial translational velocities, $\boldsymbol{u}_{i 0}^{*}=(1,0)(i=1,2,3)$ for various angles $\alpha$.

interaction between them becomes more and more powerful and drives the smallest cylinder back towards largest one, causing a second path intersection. When $\alpha>65^{\circ}$, the smallest cylinder would be driven to travel across the path of the middle-sized one and go ahead of it.

\section{Concluding remarks}

In this paper hydrodynamic interactions among many circular cylinders planartranslating in an unbounded inviscid liquid are investigated theoretically. A constructive method, which combines successive offset functions in a 'generalized cyclic permutation' manner, is presented here so as to obtain the complex potential of $N$ moving circular cylinders; the method can be extended to resolve the problem of many submerged elliptic cylinders when associated with the extended circle theorem (see Sun \& Chwang 2000) or three-dimensional bodies on condition that the pertinent harmonics are reasonably chosen. For example, for the problem of $N$ submerged spheres, solid spherical harmonics are adopted as basis sets and $Y_{n}^{m}\left(\theta_{\alpha}, \phi_{\alpha}\right) / r_{\alpha}^{n+1}(\alpha \in\{1, \ldots, N\})$ as offset functions maintaining the surface of sphere $\alpha$, where $r_{\alpha}$ denotes its radius and $Y_{n}^{m}\left(\theta_{\alpha}, \phi_{\alpha}\right)$ is a spherical harmonic function of degree $n$ and order $m$ in its body-fixed coordinates. In addition, the energy-based Lagrangian framework and momentum-type one were proved to be equivalent in the two-dimensional case. The equivalence implies that the kinetic energy $T_{f}$ expressed by (3.1) maintains a local dynamical equilibrium in the body-liquid system; the pressure integration around a submerged body is holographic, which exhibits information about accelerations of all bodies. These accelerations are coupled with one another by added masses so none of the others can be determined if some acceleration is unknown. The equivalence suggests that no matter which framework one adopts one has to solve (3.12) so as to predict the nonlinear behaviour of the cylinders under fully hydrodynamic interaction. More importantly, Galilean relativity is unsuitable for describing the submerged moving bodies under the hydrodynamic interaction.

Generally, as two circular cylinders translate side by side through a liquid, an instantaneous attractive force is exerted on them; whereas as they translate along the 


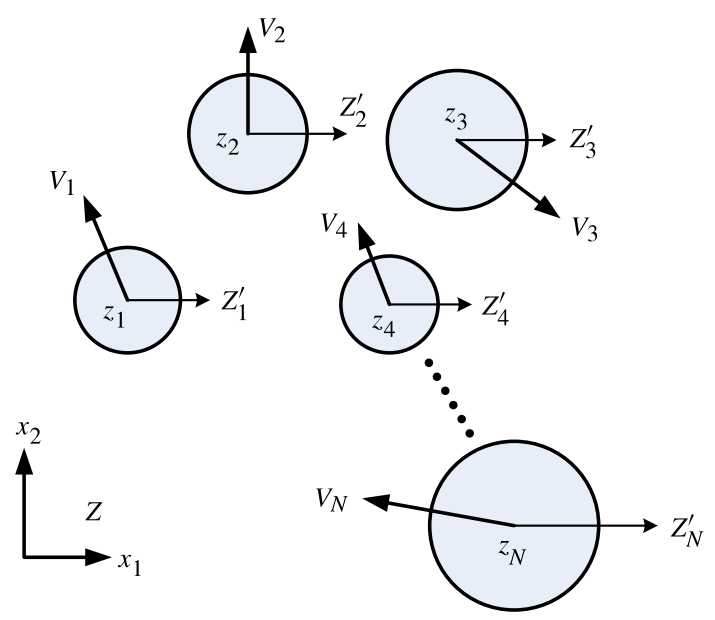

FIGURE 15. Configuration of $N$ circles and various complex planes.

line joining their centres, the instantaneous force becomes a repulsive one. But the hydrodynamic interaction in a body-liquid system of more than two bodies is not easy to predict, and it certainly leads to complicated dynamical behaviour of these bodies. For a better understanding of the behaviour, taking a case of three translational circular cylinders for example, they are classified into the several simple patterns. When three circular cylinders translate initially side by side, the stronger attractive force induced by the large cylinder drives the two smaller to approach it, resulting in an aggregating motion. As physical and kinematic parameters of the two outer cylinders are symmetric with respect to the movement of the middle one, the middle and two outer cylinders would chase after each other alternately, especially in the case that the middle cylinder is large relative to the two outer ones. When three circular cylinders translate along a line and in the same direction, repulsive forces push them away from each other. If the three cylinders are of different sizes, the middle smallest one may translate to and fro, which depends on an instantaneous resultant force induced by the other cylinders at the two sides. Other more complicated dynamical behaviours can be found through integrating several simple patterns, with which one can predict such phenomena as aggregations of the bodies, their trajectory divergences and trajectory intersections.

\section{Acknowledgements}

This work was supported in part by the NSFC (10872130) and by the HKRGC (HKU 715609E).

\section{Appendix. Solution of the Hilbert boundary-value problem}

To derive a solution satisfying boundary condition (2.3), a series of fundamental solutions first have to be constructed. For convenience, in the complex plane $Z$ the centre of every circle $\alpha(\alpha \in\{1, \ldots, N\})$ is denoted by $z_{\alpha}\left(z_{\alpha}=x_{\alpha 1}+\mathrm{i} x_{\alpha 2}\right)$, and $N$ auxiliary complex planes $Z_{\alpha}^{\prime}(\alpha=1, \ldots, N)$ are established with their origins at the corresponding $z_{\alpha}$, respectively, as shown in figure 15 .

In this situation, therefore, there are the following argument transformations:

$$
z_{\alpha}^{\prime}-z_{\beta}^{\prime}=s_{\alpha \beta} \quad(\alpha, \beta=1, \ldots, N ; \alpha \neq \beta),
$$


and separation distance relationships:

$$
z_{\alpha}-z_{\beta}=s_{\beta \alpha} \quad(\alpha, \beta=1, \ldots, N ; \alpha \neq \beta) .
$$

In order to solve the problem, a so-called generalized cyclic permutation approach is introduced in the paper. It requires that in a unidirectional cyclic process, every subsequent added singularity should be only determined by pre- $N-1$ singularities to satisfy the local boundary condition. Let us consider a basic function $w_{11 n 0}$ which expresses a translation of single circle 1 along the horizontal axis and takes the form

$$
w_{11 n 0}^{[1]}=1 / z_{1}^{\prime n} \quad(n \in N),
$$

where $N$ is the set of natural numbers and superscript [1] means that all arguments are expressed in the $Z_{1}^{\prime}$ plane. When circle 2 is put at $z_{2}$, around the circle, $w_{11 n 0}$ can be expressed in $Z_{2}^{\prime}$ as

$$
w_{11 n 0}^{[2]}=1 /\left(z_{2}^{\prime}+s_{12}\right)^{n}=\sum_{m=0}^{\infty}(-1)^{m}\left(\begin{array}{c}
m+n-1 \\
m
\end{array}\right) z_{2}^{\prime m} / s_{12}^{m+n},
$$

where $\left(\begin{array}{c}m+n-1 \\ m\end{array}\right)$ denotes a binomial coefficient. Based on the ratio test, the infinite series in (A 3) converges uniformly as $\left|z_{2}^{\prime}\right|<\left|s_{12}\right|$. The convergence domain includes a nearby zone of circle 2 . In order to ensure a constant imaginary boundary condition on the circle, an offset function $w_{11 n 1}$ should be added according to the Milne-Thomson circle theorem (see Batchelor 1967, and the reference therein),

$$
w_{11 n 1}^{[2]}=\sum_{m=1}^{\infty}(-1)^{m} H\left(m, n, R_{2}, \bar{s}_{12}\right) / z_{2}^{\prime m}=\sum_{m=1}^{\infty} \lambda_{11 m n}^{(1)} / z_{2}^{\prime m},
$$

where $H(m, n, R, s)=\left(\begin{array}{c}m+n-1 \\ m\end{array}\right) R^{2 m} / s^{m+n}$, and

$$
\lambda_{11 m n}^{(1)}=(-1)^{m} H\left(m, n, R_{2}, \bar{s}_{12}\right) \text {. }
$$

By the same criterion, the infinite series in (A $4 a$ ) converges uniformly as $\left|z_{2}^{\prime}\right|>$ $R_{2}^{2} /\left|s_{12}\right|$, which indicates that it also converges in the region outside circle 2 . When circle 3 is introduced at $z_{3}$, the sum of $w_{11 n 0}$ and $w_{11 n 1}$ around circle 3 expressed in $Z_{3}^{\prime}$ becomes

$$
\begin{aligned}
& w_{11 n 0}^{[3]}+w_{11 n 1}^{[3]}=\sum_{m=0}^{\infty}(-1)^{m} z_{3}^{\prime m}\left[\left(\begin{array}{c}
m+n-1 \\
m
\end{array}\right) / s_{13}^{m+n}+\sum_{l=1}^{\infty}\left(\begin{array}{c}
m+l-1 \\
m
\end{array}\right) \lambda_{11 l n}^{(1)} / s_{23}^{m+l}\right] \\
& \quad\left(\left|z_{3}^{\prime}\right|<\min \left\{\left|s_{13}\right|,\left|s_{23}\right|\right\}\right),
\end{aligned}
$$

which violates the boundary condition on circle 3 , and another offset function $w_{11 n 2}$ should be put into the domain so as to satisfy a constant imaginary boundary condition on the circle, that is,

$$
w_{11 n 2}^{[3]}=\sum_{m=1}^{\infty} \lambda_{11 m n}^{(2)} / z_{3}^{\prime m} \quad\left(\left|z_{3}^{\prime}\right|>\max \left\{R_{3}^{2} /\left|s_{13}\right|, R_{3}^{2} /\left|s_{23}\right|\right\}\right),
$$

where

$$
\lambda_{11 m n}^{(2)}=(-1)^{m}\left[H\left(m, n, R_{3}, \bar{s}_{13}\right)+\sum_{l=1}^{\infty} H\left(m, l, R_{3}, \bar{s}_{23}\right) \bar{\lambda}_{11 l n}^{(1)}\right] .
$$


When circle $\beta(\beta \in\{2, \ldots, N\})$ is inserted into the domain, the $(\beta-1)$ th offset function $w_{11 n, \beta-1}$ should be brought in at $z_{\beta}$ to counteract the effect of pre- $\beta-1$ singularities on its own circle. Following the same process up to the introduction of circle $N$ into the domain, the expressions for $N-1$ offset functions are derived in the form

$$
w_{11 n, \beta-1}^{[\beta]}=\sum_{m=1}^{\infty} \lambda_{11 m n}^{(\beta-1)} / z_{\beta}^{\prime m} \quad\left(\left|z_{\beta}^{\prime}\right|>\max \left\{R_{\beta}^{2} /\left|s_{1 \beta}\right|, \ldots, R_{\beta}^{2} /\left|s_{\beta-1, \beta}\right|\right\}\right)(\beta=2, \ldots, N),
$$

where

$$
\lambda_{11 m n}^{(\beta-1)}=(-1)^{m}\left[H\left(m, n, R_{\beta}, \bar{s}_{1 \beta}\right)+\sum_{l=1}^{\infty} \sum_{\alpha=1}^{\beta-2} H\left(m, l, R_{\beta}, \bar{s}_{\alpha+1, \beta}\right) \bar{\lambda}_{11 l n}^{(\alpha)}\right] .
$$

These $N-1$ offset singularities outside would break the boundary condition on circle 1 , and the corresponding offset function $w_{11 n, N}$ should be added to the domain. This means that at every cyclic step from now on, a new offset function will be added to counteract the effect of pre- $N-1$ singularities on a following circle. However, every $N$ steps will lead to cyclic variables occurring in the expressions. Therefore, a cyclic permutation function has to be introduced, which is defined as

$$
\alpha^{\dagger}=\alpha-\left[\frac{\alpha-1}{N}\right] N \quad(\alpha \in N),
$$

where $[x]$ denotes the integral part of $x$.

Repeating the above process yields an infinite sequence of offset functions, and the sum of the initial function and all offset functions sorted alternately into $N$ singularities at every $z_{\alpha}(\alpha=1, \ldots, N)$ becomes a fundamental solution to the $N$-circle problem, that is,

$$
w_{11 n}=\sum_{i=0}^{\infty} w_{11 n i}=\frac{1}{z_{1}^{\prime n}}+\sum_{\alpha=1}^{N} \sum_{m=1}^{\infty} \frac{1}{z_{\alpha}^{\prime m}} \sum_{i=0}^{\infty} \lambda_{11 m n}^{(N i+\alpha-1)} \quad(n \in N),
$$

where recurrence formulae of $\lambda_{11 m n}^{(i)}$ are

$$
\left.\begin{array}{l}
\lambda_{11 m n}^{(0)}=0, \\
\lambda_{11 m n}^{(j)}=(-1)^{m}\left[H\left(m, n, R_{j+1}, \bar{s}_{1, j+1}\right)+\sum_{l=1}^{\infty} \sum_{\alpha=1}^{j-1} H\left(m, l, R_{j+1}, \bar{s}_{\alpha+1, j+1}\right) \bar{\lambda}_{11 l n}^{(\alpha)}\right] \\
\quad(j=1, \ldots, N-1), \\
\lambda_{11 m n}^{(N i)}=(-1)^{m} \sum_{l=1}^{\infty} \sum_{\alpha=1}^{N-1} H\left(m, l, R_{1}, \bar{s}_{(\alpha+1)^{\dagger}, 1}\right) \bar{\lambda}_{11 l n}^{(N(i-1)+\alpha)}, \\
\lambda_{11 m n}^{(N i+1)}=(-1)^{m} \sum_{l=1}^{\infty} \sum_{\alpha=1}^{N-1} H\left(m, l, R_{2}, \bar{s}_{(\alpha+2)^{\dagger}, 2}\right) \bar{\lambda}_{11 l n}^{(N(i-1)+\alpha+1)}, \\
\ldots \ldots \ldots . . \\
\lambda_{11 m n}^{(N(i+1)-1)}=(-1)^{m} \sum_{l=1}^{\infty} \sum_{\alpha=1}^{N-1} H\left(m, l, R_{N}, \bar{s}_{\alpha^{\dagger}, N}\right) \bar{\lambda}_{11 l n}^{(N i+\alpha-1)} \quad(i=1, \ldots, \infty) .
\end{array}\right\}
$$


Next let us consider another basic function $w_{12 n 0}$ which expresses a translation of single circle 1 along the vertical axis and takes the form

$$
w_{12 n 0}^{[1]}=\mathrm{i} / z_{1}^{\prime n} \quad(n \in N) .
$$

By adopting the same process, the other fundamental solution to the $N$-circle problem can be derived as

$$
w_{12 n}=\sum_{i=0}^{\infty} w_{12 n i}=\mathrm{i}\left[\frac{1}{z_{1}^{\prime n}}+\sum_{\alpha=1}^{N} \sum_{m=1}^{\infty} \frac{1}{z_{\alpha}^{\prime m}} \sum_{i=0}^{\infty} \lambda_{12 m n}^{(N i+\alpha-1)}\right] \quad(n \in N),
$$

where recurrence formulae of $\lambda_{12 m n}^{(i)}$ are

$$
\left.\begin{array}{l}
\lambda_{12 m n}^{(0)}=0, \\
\lambda_{12 m n}^{(j)}=(-1)^{m+1}\left[H\left(m, n, R_{j+1}, \bar{s}_{1, j+1}\right)+\sum_{l=1}^{\infty} \sum_{\alpha=1}^{j-1} H\left(m, l, R_{j+1}, \bar{s}_{\alpha+1, j+1}\right) \bar{\lambda}_{12 l n}^{(\alpha)}\right] \\
\quad(j=1, \ldots, N-1), \\
\lambda_{12 m n}^{(N i)}=(-1)^{m+1} \sum_{l=1}^{\infty} \sum_{\alpha=1}^{N-1} H\left(m, l, R_{1}, \bar{s}_{(\alpha+1)^{\dagger}, 1}\right) \bar{\lambda}_{12 l n}^{(N(i-1)+\alpha)}, \\
\lambda_{12 m n}^{(N i+1)}=(-1)^{m+1} \sum_{l=1}^{\infty} \sum_{\alpha=1}^{N-1} H\left(m, l, R_{2}, \bar{s}_{(\alpha+2)^{\dagger}, 2}\right) \bar{\lambda}_{12 l n}^{(N(i-1)+\alpha+1)}, \\
\ldots \ldots \ldots . . \\
\lambda_{12 m n}^{(N(i+1)-1)}=(-1)^{m+1} \sum_{l=1}^{\infty} \sum_{\alpha=1}^{N-1} H\left(m, l, R_{N}, \bar{s}_{\alpha^{\dagger}, N}\right)^{(N i+\alpha-1)} \quad(i=1, \ldots, \infty) .
\end{array}\right\}
$$

As for a basic function pair which represents two translations of single circle $\alpha(\alpha \in\{1, \ldots, N\})$ along the horizontal and vertical axes respectively and takes the form $w_{\alpha 1 n 0}=1 / z_{\alpha}^{\prime n}$ and $w_{\alpha 2 n 0}=\mathrm{i} / z_{\alpha}^{\prime n}(n \in N)$, the corresponding fundamental solution pair to the $N$-circle problem can be obtained according to the cyclic substitution principle, and they are

$$
w_{\alpha 1 n}=\frac{1}{z_{\alpha}^{\prime n}}+\sum_{\gamma=1}^{N} \sum_{m=1}^{\infty} \frac{1}{z_{(\gamma+\alpha)^{\dagger}}^{\prime}} \sum_{i=0}^{\infty} \lambda_{\alpha 1 m n}^{(N i+\gamma)} \quad(\alpha \in\{1, \ldots, N\} ; n \in N),
$$


where recurrence formulae of $\lambda_{\alpha 1 m n}^{(i)}$ are

$$
\begin{aligned}
& \lambda_{\alpha 1 m n}^{(0)}=0, \\
& \lambda_{\alpha 1 m n}^{(j)}=(-1)^{m}\left[H\left(m, n, R_{(\alpha+j)^{\dagger}}, \bar{s}_{\alpha,(\alpha+j)^{\dagger}}\right)+\sum_{l=1}^{\infty} \sum_{\gamma=1}^{j-1} H\left(m, l, R_{(\alpha+j)}, \bar{s}_{(\alpha+\gamma)^{\dagger},(\alpha+j)^{\dagger}}\right) \bar{\lambda}_{\alpha 1 l n}^{(\gamma)}\right] \\
& \quad(j=1, \ldots, N-1), \\
& \lambda_{\alpha 1 m n}^{(N i)}=(-1)^{m} \sum_{l=1}^{\infty} \sum_{\gamma=1}^{N-1} H\left(m, l, R_{\alpha}, \bar{s}_{(\alpha+\gamma)^{\dagger}, \alpha}\right) \bar{\lambda}_{\alpha 1 l n}^{(N(i-1)+\gamma)}, \\
& \lambda_{\alpha 1 m n}^{(N i+1)}=(-1)^{m} \sum_{l=1}^{\infty} \sum_{\gamma=1}^{N-1} H\left(m, l, R_{(\alpha+1)^{\dagger}}, \bar{s}_{(\alpha+\gamma+1)^{\dagger},(\alpha+1)^{\dagger}}\right)^{\bar{\lambda}_{\alpha 1 l n}^{(N(i-1)+\gamma+1)},} \\
& \ldots \ldots \ldots . . . \\
& \lambda_{\alpha 1 m n}^{(N(i+1)-1)}=(-1)^{m} \sum_{l=1}^{\infty} \sum_{\gamma=1}^{N-1} H\left(m, l, R_{(\alpha+N-1)^{\dagger}}, \bar{s}_{\left.(\alpha+\gamma-1)^{\dagger},(\alpha+N-1)^{\dagger}\right)} \bar{\lambda}_{\alpha 1 l n}^{(N i+\gamma-1)}\right. \\
& \quad(i=1, \ldots, \infty),
\end{aligned}
$$

and

$$
w_{\alpha 2 n}=\mathrm{i}\left[\frac{1}{z_{\alpha}^{\prime n}}+\sum_{\gamma=1}^{N} \sum_{m=1}^{\infty} \frac{1}{z_{(\gamma+\alpha)^{\dagger}}^{\prime m}} \sum_{i=0}^{\infty} \lambda_{\alpha 2 m n}^{(N i+\gamma)}\right] \quad(\alpha \in\{1, \ldots, N\} ; n \in N),
$$

where recurrence formulae of $\lambda_{\alpha 2 m n}^{(i)}$ are

$$
\left.\begin{array}{rl}
\lambda_{\alpha 2 m n}^{(0)}= & 0, \\
\lambda_{\alpha 2 m n}^{(j)}= & (-1)^{m+1}\left[H\left(m, n, R_{(\alpha+j)^{\dagger}}, \bar{s}_{\alpha,(\alpha+j)^{\dagger}}\right)\right. \\
& \left.+\sum_{l=1}^{\infty} \sum_{\gamma=1}^{j-1} H\left(m, l, R_{(\alpha+j)^{\dagger}}, \bar{s}_{(\alpha+\gamma)^{\dagger},(\alpha+j)^{\dagger}}\right)^{-(\gamma)}{ }_{\alpha 2 l n}\right] \quad(j=1, \ldots, N-1), \\
\lambda_{\alpha 2 m n}^{(N i)}= & (-1)^{m+1} \sum_{l=1}^{\infty} \sum_{\gamma=1}^{N-1} H\left(m, l, R_{\alpha}, \bar{s}_{(\alpha+\gamma)^{\dagger}, \alpha}\right) \bar{\lambda}_{\alpha 2 l n}^{(N(i-1)+\gamma)}, \\
\lambda_{\alpha 2 m n}^{(N i+1)}= & (-1)^{m+1} \sum_{l=1}^{\infty} \sum_{\gamma=1}^{N-1} H\left(m, l, R_{(\alpha+1)^{\dagger}}, \bar{s}_{\left.(\alpha+\gamma+1)^{\dagger},(\alpha+1)^{\dagger}\right)} \bar{\lambda}_{\alpha 2 l n}^{(N(i-1)+\gamma+1)},\right. \\
\ldots \ldots . . . & \ldots \\
\lambda_{\alpha 2 m n}^{(N(i+1)-1)}=(-1)^{m+1} \sum_{l=1}^{\infty} \sum_{\gamma=1}^{N-1} H\left(m, l, R_{(\alpha+N-1)^{\dagger}}, \bar{s}_{\left.(\alpha+\gamma-1)^{\dagger},(\alpha+N-1)^{\dagger}\right)}\right) \bar{\lambda}_{\alpha 2 l n}^{(N i+\gamma-1)} \\
\quad(i=1, \ldots, \infty) .
\end{array}\right\}
$$

It should be noted that two fundamental solution sets, $w_{\alpha 1 n}$ and $w_{\alpha 2 n}(\alpha \in$ $\{1, \ldots, N\} ; n=1, \ldots, \infty)$, can be employed to construct such a solution that satisfies 
both a given boundary function on circle $\alpha$ and constant imaginary boundary conditions on the other $N-1$ circles. It means that

$$
\operatorname{Re}\left[n(z) \frac{\mathrm{d}}{\mathrm{d} z} \sum_{j=1}^{2} \sum_{n=1}^{\infty} C_{j n} w_{\alpha j n}\right]= \begin{cases}\operatorname{Re}[\bar{n}(z) V(z)] & \left(z \in c_{\alpha}\right) \\ 0 & \left(z \in c_{\beta}(\beta \neq \alpha)\right),\end{cases}
$$

where $C_{j n}(j=1,2 ; n=1, \ldots, \infty)$ are all real constants. From the uniqueness of the Fourier expansion, it is easy to prove that (A 14) holds if every $C_{j n}$ is properly chosen. Therefore, according to the superposition principle, the linear combination of all $w_{\alpha j n}(\alpha=1, \ldots, N ; j=1,2 ; n=1, \ldots, \infty)$ forms a continuous function $w(z)$,

$$
w(z)=\sum_{\alpha=1}^{N} \sum_{j=1}^{2} \sum_{n=1}^{\infty} C_{\alpha j n} w_{\alpha j n},
$$

which can satisfy the given boundary conditions on all circles, (2.3), by an appropriate choice of all real constants $C_{\alpha j n}(\alpha=1, \ldots, N ; j=1,2 ; n=1, \ldots, \infty)$.

\section{REFERENCES}

Batchelor, G. K. 1967 An Introduction to Fluid Dynamics. Cambridge University Press.

Burton, D. A., Gratus, J. \& TuCKer, R. W. 2004 Hydrodynamic forces on two moving discs. Theor. Appl. Mech. 31, 153-188.

Crowdy, D. G. 2006 Analytical solutions for uniform potential flow past multiple cylinders. Eur. J. Mech. B Fluids 25 (4), 459-470.

Crowdy, D. G. 2008 Explicit solution for the potential flow due to an assembly of stirrers in an inviscid fluid. J. Engng Maths 62 (4), 333-344.

Crowdy, D. G. 2010 A new calculus for two-dimensional vortex dynamics. Theor. Comput. Fluid Dyn. 24, 9-24.

Crowdy, D. G., Surana, A. \& Yick, K.-Y. 2007 The irrotational motion generated by two planar stirrers in inviscid fluid. Phys. Fluids 19, 018103.

Cummins, W. E. 1957 The force and moment on a body in a time-varying potential flow. J. Ship Res. 1, 1-7.

Guo, Z. \& Chwang, A. T. 1991 Oblique impact of two cylinders in a uniform flow. J. Ship Res. 35 (3), 219-229.

Hicks, W. M. 1879 On the motion of two cylinders in a fluid. Q. J. Pure Appl. Maths 16, 113-140.

LAMB, H. 1932 Hydrodynamics, 6th edn. Cambridge University Press.

LANDweber, L. \& CHWANG, A. T. 1989 Generalization of Taylor's added-mass formula for two bodies. J. Ship Res. 33 (1), 1-9.

Landweber, L., Chwang, A. T. \& Guo, Z. 1991 Interaction between two bodies translating in an inviscid fluid. J. Ship Res. 35 (1), 1-8.

LAndweber, L. \& Miloh, T. 1980 Unsteady Lagally theorem for multipoles and deformable bodies. J. Fluid Mech. 96, 33-46.

LANDWEBER, L. \& YiH, C. S. 1956 Forces, moments, and added masses for Rankine bodies. J. Fluid Mech. 1, 319-336.

Milne-Thomson, L. M. 1960 Theoretical Hydrodynamics, 4th edn. Macmillan.

Miloh, T. \& LANDweber, L. 1981 Generalization of the Kelvin-Kirchhoff equations for the motion of a body through a fluid. Phys. Fluids 24 (1), 6-9.

NAIR, S. \& KANSO, E. 2007 Hydrodynamically coupled rigid bodies. J. Fluid Mech. 592, 393-411.

SUN, R. \& CHWANG, A. T. 2000 Hydrodynamic interaction between two cylinders with rotation. J. Phys. Soc Japan 70 (1), 91-102.

Sun, R. \& CHWAnG, A. T. 2006 Interaction of a floating elliptic cylinder with a vibrating circular cylinder. J. Hydrodyn. B 18 (4), 481-491. 
TAYLOR, G. I. 1928 The energy of a body moving in an infinite fluid, with an application to airships. Proc. R. Soc.Lond. A 120, 13-21.

Tchieu, A. A., Crowdy, D. G. \& Leonard, A. 2010 Fluid-structure interaction of two bodies in an inviscid fluid. Phys. Fluids 22, 107101.

WANG, Q. X. 2004 Interaction of two circular cylinders in inviscid fluid. Phys. Fluids 16, 4412-4425.

WANG, Q. X. 2007 An analytical solution for two slender bodies of revolution translating in very close proximity. J. Fluid Mech. 582, 223-251.

WANG, Q. X. 2005 Analysis of a slender body moving near a curved-ground. Phys. Fluids 17, 097102.

Yamamoto, T. 1976 Hydrodynamic forces on multiple circular cylinders. ASCE J. Hydraul. Div. 102 (HY9), 1193-1210. 